\title{
Selbstbiographie des Stadtpfarrers Wolfgang Ammon von Marktbreit († I634).
}

Mitgeteilt von FRANZ HÜTTNER.

In der Würzburger Universitätsbibliothek befindet sich als M. ch. f. 440 eine aus dem Jahre 1727 stammende Abschrift der Selbstbiographie oder Hauschronik des protestantischen Pfarrers Wolfgang Ammon zu Marktbreit, einer am Main im bayerischen Bezirksamt Kitzingen gelegenen Stadt, deren Geschichte im Jahre 1864 Pfarrer Plochmann geschrieben hat. Das Manuskript besteht aus 51 Folioblättern; am Rande sind die Folien des Originals angegeben.

Da diese Hauschronik viele kleine interessante Züge enthält, dürfte sie sich zur Veröffentlichung eignen. Der Verfasser schildert in derselben das Leben seines gleichnamigen Vaters, welcher $1540 \mathrm{zu}$ Elsa bei Coburg geboren wurde und 1589 als Pfarrer in Marktbreit starb, das Leben seiner Mutter und übrigen Verwandten, sowie seine eigenen Schicksale bis zum Jahre 1633. Bei der Plünderung und Verwüstung Marktbreits durch die Soldaten Piccolominis 1634 wurde er misshandelt und starb an den Folgen dieser Misshandlungen kurze Zeit darauf, am 22. September 1634. Von späterer Hand stammen Zusätze, welche in die Abschrift aufgenommen wurden. 
[B1. 1] Wolfgang Ammons, gewesenen Caplans, dann Stadtparrers allhier, Hausschronica.

Hauss-Chronica oder Geschichtbuch, was sich im Ammonischen Geschlecht denkwürdiges zugitragen, von etlichen Jahren her, sampt andern Verlauffungen, so viel ich notirt hab; dann ich nicht allemal gleich fleissig gewesen. Beschrieben und renovirt von, Wolfgang Ammonio dem jüngern, Mitd ienern am Wort Gottes zu Markibrait, der damals ins 54. Jahr gieng anno domini 1625. Psalm 119 vers. 9. Ich hab alles dings ein Encle gesehen, aber dein Gebot währet.

[fol.3.] Im Namen desdreyeinigen meines lieben Gottes, Vaters, Sones und heiligen Geistes, hochgelobet in Ewigkeit. Will ich, der Autor oder Schreiber diess Buchs Anfang machen und nehmen von meinem lieben Vattern, als von deme nāchst Gott ich das Leben habe. So ist nun gemeldter mein Vater $(M$. Wolfg. Apımonius) zu Elsa geboren, welches ein Dorff ist, in Coburgischer Herrschafft gelegen, ein Viertelmeil von Rothach oder grosse Meil von Coburg gelegen im Grabfeld, Anno 1540. Sein Vater hat geheissen Jacob Ammon, sein Anherr Georg Ammon, sein Anfrau Christina, der Geburt eine Reumannin, sein Mutter Catharina (deren Vatter Claus Kob und Catharina die Mutter eine Blümingin von Geschlecht), wie solches alles sein Geburtsbrief Anno 1587 den 14. Decembris zu gemeldten Rotha datirt, ausweiset.

(In demselben stehen unter andern diese Wort: $\mathrm{Er}$ habe von seinen 4 Anherrn hero keinen Schäffer, Schinder, Schollern, Schergen, Bütel, Pfeiffer, Bader, Badstuber). In der h. Tauff ist ihm der Name Wolfgangi [fol. 3] gegeben worden.

Als er nun verständig worden oder zur Schulen gehen können, ist er täglich gen Rotha, meines Behaltens, in die Schul gangen, uff 3 Jahr lang, mittags drinnen blieben .[Bl. 2'] und mit Eyern sich beholfen, hernach gen Coburg in die Kost verliehen und da studirt mit Matthaeo Hecklino, so mit der Zeit Pfarrer zu Dornheim worden. In seiner Jugend Anno 1560 hat er einen bösen Schenkel bekommen und von einem Bauersmann sich wieder curiren lassen im Dorf Horb [östlich von Sonnenfeld], der einen guten Wundarzt geben und ihm die Hüfft eingerichtet. Ist dem- 
nach bey der Mutter daheim geblieben und 2 Monat vom Studirn abgehalten worden.

Eben in diesem 1560. Jahr und im Monat Majo ist er, nach verlassener Coburgischer Schul gen $H$ of ins Voitland kommen mit Johanne Wolfio und [fol. 4] Andrea Zwingero und an $M$. Johannem Streitberger ${ }^{1}$ ), zum Hof-Pfarrern und Superintendenten [darüber steht Collegam scholae meines Wissens]") (so hernach Doctor worden und Superint. zu Culmbach), ron M. Nicolao Culmbacher verschrieben, ein armer Schüler gewesen, umbs Brod mitgesungen, ein Vettern Georg Helmut da gehabt.

Seine praeceptores waren: $M$. Johann Streitberger, Jacob Schlemmer, Laurentius Codmann (dess Salomonis Codomanni des āltern zu Kitzingen, weiland Decani [er war dort Dekan 1598-1622] Vatter), welcher hernach ein Pfarrer und lezlich Superintendent zu Schönfeld, M. Christoph Cadesreuter (der auch mein Rector gewesen). Aber er hat sich, inmassen ers selbsten hernach so schrifftlich so mündlich beklagt, seine Gesellschaft lassen bereden und gen Dressden kommen mit obgedachten Gefehrden, allda er Tobiam Mōstelium Rectorem gehōrt, und ihm wegen der Poeterey lieb gewesen. Anno 1561 am andern Ostertag ist er mit Zwingero weggezogen und gen Altenburg kommen, hernach in Bōhmen zum warmen Bad mit Mezlero, Cantore Aldenburgico, gezogen, und haben 4 Tag gebadet. Anno 1561 13. Octobr. gen Wittenberg kommen und sich deponiren ${ }^{3}$ ) lassen mit seinem Gesellen Medlero, Johann Talhamer Beyren, Wolfgang Möstel von der Weiden [Weiden, Stadt in der Oberpfalz], des Rectors zu Dressden

7) $V_{g l}$. über ihn Beiträge zur bayerischen Kirchengeschichte, her. v. D. Theodor Kolde 2, 39 Anm. 1:

Mag. Joh. Streitberger aus Hof, studierte in Wittenberg (inscrib. Sommersem. 1532, Förstem. 146; Baccalaureus 1538, Köstlin III, 1), seit 1538 Diakon an St. Wenzel in Naumburg, an Medlers Kirche, dessen Schwiegersohn er wurde; wurde 9. Febr. 1542 in Wittenberg Magister; 1545 folgte er seinem Sch wiegervater nach Braunschweig und starb 10. April 1602, 85 Jahre alt, als Pfarrer und Generalsuperintendent in Culmbach.

2) Vgl. ferner 4, 71 Anm. 1. Superintendent in Hof 1552-1567. Vgl. noch Fickenscher, Gel. Fürstent. Baireut, Bd. IX, 89-9j, woselbst auch die ältere Literatur berūcksichtigt ist. Dazu die Biographie Streitbergers von Hagen in -Archiv f. Gesch. u. Altertumsk, v. Oberfr. VI. Bd. 2. Heft. 1855.

\%) Beitr. II1, 138, Anm. 5: Die Deposition hiess die Feierlichkeit, durch welthe die neu angekommenen Siudenten unter allerlei, zum Teil lächerlichen Ceremorien von einem dazu bestellten Depositor in die Würde der akademischen Bürger aufgenommen vurden. 
Brudern. Ist ins Studentenbuch oder Matricul eingeschrieben von [B1. 3] Herrn Doctore Georgio Majore, der h. Schrifft Professore, vermög habenden Zeugniss. Diesser damals Rector hat Ihme, meinem Vattern eingerathen, er soll sich nicht mehr Ammonem in tertia Declinatione schreiben, sondern Ammonium in secunda, dem ist bissher gefolget worden.

Anno 62 24. Sept., dieweil er die Lufft zu Wittenberg nit vertragen können, da schwach worden und clistirt worden, kommt er gen Jena, mit Johann Veit, Diacono Ummerstadiensi, wird darauf in die Matricul einverleibt, den 25. Sept. von Herrn Doctor Johann Schröter, Medico und Rectore. Anno 1564 zeucht er wiederum nach Wittenberg mit Georgio Fabro, kommt an den 20. Oct.

Anno 1565 23. Aug. wird er Magister zu Wittenberg und hat unter 21 den 13. locum. Caesius, der Calendermacher, hat den 12. Besiehe das Testimonium, so schön [fol. 7].

Die Namen deren, so mit meinem Vatter Magistri worden, unter dem Decano $M$. Wolfgango Zeisero Fuldensi Wittenbergae. 1. Andreas Frankenberger Memingensis. 2. Johannes Fleischner Vratislaviensis. 3. Johannes Bochmannus Jenensis. 4. Georgius Cunchius Pomeranus. 5. Jacobus Soldner Glauchensis. 6. Jacobus Rolchius Peirnensis. 7. Reinerus Reineck Steinhemius. 8. Georǵius Spalatinus Arnstadensis. 9. Johannes Crato Benconensis. 10. Gottfridus Rechter Haflensis. 11. Caspar Ludovicus Hammonensis. 12. Georgius Caesius Rotenburgensis. 13. Wolfgangus Ammonius Coburgensis. 14. Vitus Soldin Gemingensis. 15. Samuel Wincklerus Torgensis. 16. Justus Mollerus Hamelensis. 17. Johannes Nuberus Hilpersteinensis. 18. Paulus Peter Alburgensis. 19. Rupertus Wilsius Cygneus. 20. Philippus Klinger Tutterstadiensis. 21. Andreas Laborator Carinthius.

[Bl. 3'.] Anno 1566 3. Novembr. wird er zur Pfarr Weidelbach [im Amtsgericht Dinkelsbühl], so marggräfisch worden, als der 1. evangelische Pfarrer daselbst, zu Onolzbach ordinirt von nachfolgenden Kirchendienern: Herrn Georgio Kargen, Johanne Baptista Lechelio, Johanne Unfugio, Georgio Grenner, Georgio Kellin, Johanne Scopio, Wolfg. Fröschel; und hat der Herr Kargius das Testimonium eigenhändig geschrieben. 
[fol. 5] Anno 1567 10. Martii, Montags nach Mitfasten, hält er Verlöbniss mit meiner Mutter Maria, Herrn M. Wolffgangi Jungens, reformirten Stifftsdechants [1563 das Stift aufgehoben] zu Feuchtwangen ledigen Tocliter; copulirt sie biss auf fernere Priestershand M. Balthasar Hillenmeyr, Prediger und Superintendens zu Feuchtwang.

Anno 1567 22. April, Dienstag nach Jubilate, haben sie ihr hochzeitich Fest celebrirt. Brautführer sind gewesen N1. Johann Tettelbach und $M$. Johann Göłz, hat sie zusammen gegeben H. Simon Priester.

Epithalamium oder Hochzeitvers hat ihnen geschrieben, so ich gedruckt fürweisen kan, $M$. Johann Schirmer, derzeit Pfarrer zu Feuchtwangen, so hernach gen Kitzingen kommen und im Wasser ertrunken.

Die Jahrzahl stehet auch in diesen Versen:

VergILIls ortls ChrIsto LVX aVsplCe fVLsit;

JVnCta VbI Volfgango VIrgo Marla VIro.

Anno 1567 2. Dec. hat er die Pfarr Weidelbach, weil er gern studiren wollen und im Dorf nit Gelegenheit gehabt, mit Rath seines $\mathrm{H}$. Schwehers selbsten resignirt und den 7 . dito ein gut Testimonium bekommen von Friederich Alexandern von Seckendorff, Marggräfischen seinem Amptnann, und Herrn Balthasaro Hillemcyern, seinem Superintendenten, den 30. Dec. noch ein Zeugniss erhoben, vom teutschherrischen Vogt Jacob Hartmann zu Dünckeisbühe!, der ihm die pfärrliche Einkommen gereicht bissher. Darauf zu Dünkelsbühel der evangelischen Kirchen Helfer oder Diaconus worden und blieben 11 Jahr 6 Monat bei $H$. Johann Knauern und M. Thoma Venatorio.

Er hat mit meiner Mutter gezeugt nachfolgende Kinder: Anno 1568 29. April Donnerstag zwene Zwilling geborn, I. das eine Kind Samuel genannt worden und aus der Tauff gehoben von besagtem Herrn M. Johanne Knauer, Neuburgischen geliehenen Pfarrern zu Dinckelsbühl.

II. Das ander ist Catharina, nach seiner Fran Doden Catharina Drechslin, einer Geschlechterin, genannt. Sind von Georgio Stifftenbergern, Pfarrer zu Segering [Segringen im Amtsgericht Dinkelsbühl], getaufft und beede verschieden. 
III. Anno 1570 19. Jan. Donnerstag um 2 in der Nacht ist Catharina, die andere diess Namens geborn, darauf des Tags um 7 hor von Herrn M. Johanne Knauer getaufit, von vorbesagter Drechslin gehoben. Der Schwehervatter, als ihm solches referirt, giebt dem Botten über den Lohn 6 Xr zum Bottenbrod. [fol. 6] Da diess Kind noch nicht zwey Jahr alt, verscheidets Anno 1571 18. Aug. Abends um 6 Hor. Die Jahrzahl stehet in diesem Vers:

Nata MIhI LVDens oblit KatharIna sVaVIs.

IV. Anno 1572 7. Jan. bin ich Wolfgangus Ammonius geborn, davon an seinem Ort.

V. Anno 1573 6. Novemb. Freitag Daniel nach 1 Uhr in der Nacht, M. Knauer taufft ihn, Daniel Teller, ein Kaufherr, hebt ihn, welcher den 5. Martii Anno 74 verschieden, Freitags, seines Alters in der 17. Wochen.

VI. Anno 1577 23. April, Dienstag nach Misericordias, Maria Catharina geborn, Abends zwischen 5 und 6 . Tauff sie Herr Stiffenberger, Pfarrer im Closter Roth. Hebt sie aus der Tauff Frau Catharina Drechslin.

VII. Anno 1579 10. Maji Sontag Jubilate frühe ein wenig nach 3 Hor, im Zeichen Scorpion, Euphrosyna. Taufft sie Johannes Salzer, der neue Caplan oder Helfer, hebt sie Frau Sibylla, Herrn Doctor Lucae Beckers, Rechtsgelehrten und Beysitzers im Cammergericht, Haussfrau. Ist verschieden 23. Julii diess Jahrs zu Markbrait, frühe um $4 \mathrm{im}$ Neumond (als sie gelebt 10 Wochen 3 Tag).

VIII. Anno 1581 1. Julii Samstags Sophia um 3 Hor gegen Abend zu Markbreit geborn, durch Adam Angermann Capellan [Plochmann, Gesch. v. Marktbreit S. 99] getaufft, hebt sie aus der Tauff Herrn Johann Orts, Schuldheissens Haussfrau, verscheidet Anno 83 21. Sept

[BI. 4'] IX. Anno 1583 Georg Ludwig den 27. Jul. Abends zwischen 10 und 11 Samstags im Zeichen $)$. Taufft ihn folgenden Tags um 4 Adam Angermann, hebt ihn Matthes Jahn, Vogt anstatt des wolgebornen Herrn Georg Ludwig von Seinssheim Freyherrns [geboren 26. Jan. 1514, in den Freiherrnstand erhoben 15. März 1580]. Von diessem Sohn stehet mehr unten. 
X. Anno 1586 26. April frühe zwischen 4 und 5 Barbara, Dienstags nach Jubilate, an S. Albertstag (an welchen Tag eben vor 19 Jahren die Eltern Hochzeit gemacht zu Feuchtwangen). Ist aus der Tauff gehoben von wolehrn gedachts Herrns von Sainssheim Gemahlin Barbara von Hessberg in der Person. Von dieser Tochter stehet mehr unten.

[fol. 8.] So ist nun mein lieber Vatter Ammonius ein recht gelehrter Mann gewesen, wie er von vielen Gelehrten das Zeugniss hat, ist ein guter Poet [Plochmann, Gesch. v. Marktbreit S. 88], wie sein lateinisches Gesangbuch, Odae ecclesiasticae genannt, ausweiset (welches er Anno 1570 angefangen zu machen); auch ein trefflicher Linguist und Sprachenkundig gewesen, deme der alte Herr $\mathrm{H}$. Georg Ludwig von Seinssheim die grosse Kōnigliche Bibel mit vielen Sprachen zu Ehren erkaufft und geliehen; auch ist ihm die Profession der hebraeischen Sprach zu Jena angeboten worden, welche er aber abgeschlagen wegen seiner angesponnenen Wassersucht etc.

Anno 1579 ist er den 10. Julii mit seinen Weib und Kindern gen Marktbreit als Pfarrer ufgezogen, nachdem er zuvor seine Probpredigt am andern Pfingsttag da gehalten und mit vielen ansehenlichen commendationibus ausgerüstet gewesen, die beste aber bey. sich selbst gehabt im Busen.

Den 9. Julii uffgebrochen von dem Ort, da er 10 Jahr und 6 Monat der Kirche gedienet. Hat sich als einen eiferigen Lehrer erwiesen, aber bey vielen seiner Zuhörern und Collegis schlechten Dank verdient. Doctor Ruprechten, der bey 3 Jahren nicht hie communicirt, nit [BI. 5] wollen zu Gevatter stehen lassen und darum mit ihm rechten müssen, welches die alte Frau von Seinssheim [geb. v. Hessberg], ein Gevatterin, verlegt, doch endlich gewonnen und gemeldten Doctor aus dem Flecken bracht.

Anno 83 3. April ein schön Wappen, wie der Brief ausweiset, erlanget, von Paulo Melisso, Comite Palatino. Darüber er mein Vatter (seliger) diese Vers weiland gemacht:

In Insignia M. Wolfg. Ammonii Elsani.

Prima manus manui juncta et quando, auspice Christo

Adducta est primo femina prima viro.

Namque Evae dextram dextrae commisit Adami 
Et dextrae sacrum pondus habere dedit.

Haec ait et fidei servandae tessera firma

Et certum veri pignus amoris erit.

[fol. 9.] Dextra igitur dextrae connexa antiqua parentum

In paradisiaco signa fuere statu.

Talia et Ammoniae sunt clara insignia gentis,

Nimirum dextrae dextera juncta manus.

Que rapta sunt firmae fidei monumenta tenendae

Et larga gentis de pietate monent.

Christe, fidem semper pia gens tibi servet; alumnum Alme tua dextra protege, conde, fove.

Daraus dann sein gottesfürchtiges lieb-, ehr- und tugendreiches Herz zu sehen; aber mehr nicht, damit ich der Sachen nicht zu viel gethun haben scheine.

Anno 88 26. Jun. hat er ihm ein Fontanell an dem rechten Schenkel sezen lassen und alle Tag ein Erbs in das Loch gelegt. Den 9. Sept. diess Jahrs noch ein Loch in den andern Schenkel machen lassen, weil die Geschwulst und Wassersucht ihm heftig zugesetzt.

Anno 1589 26. Januar ist er in wahrer Anruffung und Bekenntniss Jesu Christi entschlaffen, alt 49 Jahr. M. Nicol. Bauch, sein Collega [von Eibelstadt, Pfarrer 1589-1597], hat die Leichpredigt gehalten. Der gar alte Herr von Seinssheim [geboren $1514,+1591]$ ist persōnlich dabei gewesen und hat geweint, dass ihm sein sammets Häublein vom Haupt herabgefallen, hat auch diese Wort verlauten lassen: Ich hab wol Sorg, ich bekomme mein Lebtag keinen solchen Pfarrer mehr.

[Bl. 5', fol. 10] Es wird mehr gemeldtes meines Vatters sel. auch ehrlich gedacht in vielen Büchern, sonderlich in vita illustris et generosi herois Domini Georgii Ludovici a Seinsheim; in seinen Odis gedenkt Trostius hinden im Appendice auch gar herrlich etc.

Es haben seine liebe Eltern Jacob Amman (der ein Bauer und feines Vermögens gewesen) und Catharina, seine Mutter (die über 100 Jahr alt worden, ein recht gottselig Weib und den armen Leuten viel guts gethan, sie jährlich uff der Kirben gespeist, in der Scheuren, etlich Tisch voll, und darum, unter anderer Gefertschafft, von ihnen mit Hauffen begleitet und beweinet als eine 
rechte Tabea, Anno 90 im Januario), diese seine liebe Eltern haben sonst mehr Kinder gehabt; meines Vattern sel. Schwester hab ich gesehen, da sie bey 60 Jahren alt gewesen. Der eine sein Bruder hat geheissen Herr Lorenz Ammon, Pfarrer zu Elsa und Baterstat, dieses sein Weib, meines Vettern Hanssen Ammans (Burgermeisters weiland zu Helpurg) Mutter ist gestorben Anno 1560. Der zweite sein Bruder Georg Anno 6S 7. Jan. Hochzeit gemacht. Der dritte, Hanss, der Bauer, hat Anno 62 20. Nov. Hochzeit gehalten, ist päbstisch gewesen und den 23. Martii verschieden Anno 71, und welches meinem Vatter selig sehr zu Herzen gangen, sind ihm die 3 Brüder in einem Jahr verschieden, wenig Tag nacheinander; der Herr Lorenz (welcher viel bey meinem Vatter seligen gethan, Geld hergeliehen zu seinen studiis, ihn an Stöffelium, Professorem zu Jena, verschrieben etc.) den 27. Jun. Anno 1571. Er hat ihnen auch solches Epitaphium gemacht, darinnen die Jahrzahl stehet:

post blnos pastor LaVrentlVs, antea fratres

terrae ManDatos tertlVs Ipse oblit.

[Bl. 6, fol. 11.]

Meiner Mutter Leben.

Sie ist gebohrn zu Feuchtwangen in einer marggräfischen Brandenburgischen, 1 Meil von Dinkeisbühel gelegenen Stadt (so weiland eine Reichsstadt gewesen) Anno 1546 30. Januar Nachmittag um 3 Hor. Aus der h. Tauff gehoben von der Edlen Frauen Helena, Jungkher Adams von Elrichshausen zum Dürrenhof Hausehr, die eine Tochter Melchior Senfts gewesen, eine erstgebohrne Tochter ihres Vatters. Ihr Vatter ist gewesen Herr M. Wolfg. Jung, des reformirten Stiffts daselbsten lezter Dechant, so auch geprediget, doch nicht offt. Sein Bruder Herr Martin Jung, Hauptmann im Türkenkrieg, unter dem Grafen von Serin, dessen Wolfsbelz er bekommen und getragen, auch ein Marschalk Herrn Georg Friderichen, Marggrafens zu Brandenburg, stirbt Anno 1575 23. Oct. seines Alters im 64. Jahr.

Mein Anherr aber, M. Wolfg. Jung, ist eines Schreiners Sohn, meines Behaltens, gewesen, dessen Vatter in der Kirchen, wenn man von Rotenburg hinein zeucht, nit weit vom Feucht- 
wanger Thor viel gearbeitet. Er ist zu Onolzbach bey einem Domherrn als ein Vetter gar hart erzogen worden.

lhre Mutter ist gewesen Sara, Herrn M. Bernhardi Wurzelmanns, ') Pfarrers zu Dinkelsbühel und Weildingischen [Weiltingen, Markt im B. A. Dinke!sbühl] Capiteis Decani Tochter. (Besiehe die Vorrede meines Vatters seligen über sein publicirtes lateinisch uind teutsches Gesangbuch). Sie ist gestorben im Januario Anno 1572. (1. potius, ut p. 15 legitur, Anno 1587 die 10. Maji.)

Ehe ich weiter fortfahre, wiil ich zuror dieser Sarae, meiner Anfrau, ihre Ankunfft und Geschlecht weiter beschreiben.

\section{[Bi. 0 , fol. 12] Genealogia familiae ab antiqua stirpe Wurzelmannorum.}

Der alte N. Wurzelmann ein Beck gewesen zu Heilbronn, einer Reichsstadt am Neckar, da er auch begraben liegt, Catharina, seine Haussfrau, hat verlassen 3 Söhne und 2 Töchter, welcher Nahmen samt den verlassenen Kindern sind diese:

\section{Sōhne:}

1. M. Bernhardt, Pfarrer zu Dünkelsbühel, seine Haussfrau Margareta, hat verlassen:

Philipp, hat zu Strassburg bey Bucero²) studirt und ist ledig gestorben.

Sara, M. Wolfg. Jungen, Stifftsdechants zu Feuchtwangen Haussfrau, hat verlassen 4 Söhn: Adam, Abraham, Philipp, Paul

1) V'gl. Beitr, 2, 301, Anm. Ritzander, Wurzelmann, Sohn des Bürgermeisters in Wimpfen, Schwager Erhard Sclnepifs, dessen Gattin seine Schwester war (vgl. Hartmann, Schnepff, 1870 S. 9), war zuerst Kanonikus im Stift zu Wimpfen, welche Stelle er aber aufgab, weil er das Evangelium nicht predigen durfte; er ward darauf Pfarrer in Schwaigem im Kraichgau und 1534, von Brenz und Adam Weiss in Krailsheim empfohlen, I'farrer zu Dinkelsbühl (vgl. Bossert, Briefe und Akten zur Gesch. der frānk. Rcform. in Theol. Studien aus Württemberg VII, $13 \mathrm{ff}$.). Nach des Kaisers Sieg bei Giengen 1546 musste Dinkelsbühl das Interim annehmen und der betagte Mann wurde 1549 Katechist in Benningen in Württenberg, Bossert, Interim in Württemb. (Schriften des V. f. Rfgesch. No. 42 H. 46 S. 113).

Beitr. 5, 197 Anm. Bernhard Wurzelmann, der Sohn des Būrgermeisters in W'impfen, sein Bruder war 1533 Stadtschreiber in Schwäbisch-Hall, studierte in Heidelberg wo er sich im Frühjahr 1512 den Magistergrad erwarb. Im Dez. 1533 trat er die Stelle als Pfarrer in Dinkelsbühl an und schaffte am 5. Januar 1534 die Messe in der Gecrgskirche ab (Steichele, Das Bistum Augsburg 3, 250, der ihn übrigens fälschlich schon 1544 sterben lăsst).

2) Vgl. Beilr. 1, $134 \mathrm{f}$. Butzer hatte in Heidelberg studiert, und in Strassburg sassen die Studierenden der Tlıcologie aus der Pfalz zu seinen Füssen. cf. Rōhrich, Gesch. d. Reformation des Elsasses II, 240; Gelbert, Bader 204 ff. Nach Einführung des Interims $20 \mathrm{~g}$ er nach London und starb dort am 28 . Februar 1552. 
und 5 Töchter: Mariam, M. Wolfg. Ammonii Weib; Justinam, Mariam Jacobi, Mariam Magdalenam, Euphrosynam, so Anno 1570 im Majo am Stein gestorben.

Catharina, Herm Lorenzen, Pfarrers zu Binningen am Neckar, Weib.

Anna, hat Herrn Michel Bierdūmpfel, Pfarrer zu Belsenberg, gehabt und 1 Sohn, Daniel, verlassen.

II. Dieterich, Goldschmiedt und Pfeningmeister im Türkenkrieg, hat 5 Töchter verlassen.

1. An na, hat M. Joh. Mercklinum von Kauffbeuern, Victorini Vettern.

2. Apolloniam, hat Herrn Joh. Faust, Pfarrern zu Nehren im Würtembergischen.

3. Catharinam, M. Georg Wegmanns, Pfarrers zu Cassel in der obern Pfalz, hat eine Tochter verlassen, Susanna.

4. Christinam, Herrn Sebastian Mokels, Pfarrers zu Offterdingen, und

5. Barbaram, Herrn Michel Kisers, Pfarrers zu Eizingen im Würtembergischen, Eheweiber.

III. Matern, Stadtschreiber zu Schwäbischen Hall, hat verlassen:

Matthes, ein Kriegsmann, des Planken Mutter Bruder.

Anna, Dieterich Planken, Spitalschreibers zu Hall, Haussfrau, hat verlassen 2 Söhn:

1. Michel, Vogt zu Mur [Altenmuhr] bei Gunzenhaussen.

2. Dieterich, hat studirt und 2 Töchter a) Anna, b) Maria, hat einen Capellan zu Creilsheim gehabt.

Elisabeth, Stadtschrciberin zum Hirschhorn am Neckar, ohne Kinder gestorben.

Närrin zu Ingolstadt im Spital gestorben.

Töchter:

Apollonia, hat 2 Männer gehabt: 1. Son Claus zu Wimpfen, mit dem erzeugt:

a) Claus, ein Soldat.

b) Apollonia, Wendel Hiplers, Doctors, Haussfrau, hat verlassen 2 Söhne a) Wendel, Doctor juris zu Tübingen, b) Johann, Amptmann zum Hirschhorn. 
[Vgl. Hartmann, Erhard Schnepff, 1870. S. 150.]

2. Doctor Ehinger, mit dem erzeugt eine Tochter Maria, hat einen Weingartner zu Heilbronn.

Margareta, Doctor Erhard Schnepfen, ") Uul. Hartmann, Erhard Schnepf, Tüb. 1870], Professors und Pfarrers zu Jena Haussfrau [er wurde im Sommer 1549 Professor in Jena und dort am 2. November 1558 begraben], hat verlassen 4 Söhne mit Nahmen:

1. Dieterich (Theodoric) Schnepf, Doct., Prof. und Pfarrer zu Tübingen [vgl. Hartmann S. 73], hat einen Sohn zu Eisenach, Doct juris.

2. Joh. Erhard, Secretar zu Coburg.

3. Eusebius, Procurator zu Heilbronn.

4. Daniel, ein sāchsischer Stipendiarius, und 1 Tochter Blandina, hat 2 Mānner gehabt: 1. M. Victorin Strigelium [Professor in Jena, cf. Hartmann S. 71], ohne Kinder; 2. Johann Fetscher, D. Georg Hambergers Weibs Bruder.

[B1. 7', fol. 14] Nun will ich von meiner Mutter selig Geschwistrigten einen kurzen Bericht thun, so viel ich weiss, darnach ihr Leben fortmachen.

Adam Jung ist Doctor der Arzney worden zu Tübingen, 18. Febr. Anno 1581, hat meinem seel. Vatter auf seine Hochzeit Vers gemacht, wie auch in sein Gesangbuch, die dabey gedrukkt.

Abraham ist ein Pfarrer zu Dorf Güting lange Zeit gewesen, guter Linguist und daselbst [in Dorfgütingen, Amtsgerichts Feuchtwangen] gestorben Anno 1624; sein erstes Weib Magdalena starb 1582, die $z$ weite Ottilia, meiner ersten Frauen Stieffschwester, Herr Hansen Cuppelichs Tochter, davon unten fol. 27.

Philippus ist Magister und guter Hebraeus, Capellan zu Unterschwaningen [im Amtsgericht Wassertrūdingen], Pfarrer zu Lehngūting [Lehengütingen im Amtsgericht Dinkelsbühl] und lezlich Pfarrer zu Leutershaussen [Stadt im Amtsgericht Ansbach] worden.

Dessen erster Sohn, M. Thomas Jung, Pfarrer zu Mönchsondtheim [im Amtsgericht Scheinfeld] seiter Anno 1614. 2. Georg

1) Vgl. Beitr. 3, 134, Anm. 3. Erh. Schnepf war seit 1. Februer 1544 Professor der Theologie und Pfarrer in Tübingen, vgl. Hartmann, Schnepf, Tüb. 1870, S. 60. 
Friderich Jung, Conrector zu Onolzbach, dann Rector und Adjunctus bey der Pfarrkirchen seit Anno 59.

Paulus hat sich der Schreiberey beflissen in der Jugend im Closter Lorch zu Würtenberg und anderswo, lezlich viel Jahr zu Onolzbach Kornschreiber gewesen, Anno 1593 10. Jul. mit seiner Agatha Hochzeit gemacht (sein Aidam ist Herr Stubenfal, des Raths zu Onolzbach, wo künstliche Dekk machet). Ich bin uff der Hochzeit gewesen und hab ein Epithalamium oder Vers gemacht.

Justina hat Herrn Georgium Fischer, Pfarrern zu Schopfloch [im Amtsgericht Dinkelsbühl], der lezlich gen Hammersheim [Hemmersheim im Amtsgericht Uffenheim] und Gülchsheim [im Amtsgericht Uffenheim] zum Pfarrer promoviret worden, gehabt.

Maria Jacobi hat einen Handelsmann zu Kirch an der Teck im Würtenberger Land bekommen.

Maria Magdalena ist uff Beförderung obgedachten Doctors stattlich ankommen und hat einen Moser, so fast ein Edelmannsgut und höchsten Dienst zu Göppingen gehabt, zur Ehe bekommen. Hernach einen andern Mann gehabt, welcher ein Soldat gew'esen, sie nicht gleich dem ersten gehalten.

Euphrosyna, $\dagger$ anno 1570.

Bl. 8, fol. 15] So sind nun meiner lieben Mutter selig Eltern, M. Wolfg. Jung (welcher Anno 1575 20. Dec. 2 Monat und 2 Tag nach seinem Bruder Martino, Hauptmann, verschieden, an einem viertägigen Feber, so er 13 Wochen gehabt, seines Alters im 58. Jahr) und Sara, vom Geschlecht eine Wurzelmännin (welche Anno 1587 10. Maji verschieden an der Wassersucht) und haben ein ehrliches Vermögen hinderlassen. Vid. Epitaph. in der Stifftskirchen zu Feuchtwang im Chor.

Diese Eltern haben ihre Tochter, meine Mutter, zum Catechismo und allen weiblichen Tugenden ganz ernstlich mit Worten und guten Exempel erzogen, immassen sie dann des Kargii Fragstück im Catechismo, die damals nicht alt gewesen, von Wort zu Wort, auch in Alter erzehlen können guten Theils.

Ihre Verlöbniss betreffend mit meinem sel. Vatter stehet oben im 5. Blat, wie auch die Hochzeit und Kinder daselbst und folgends zu befinden. 
Als sie 3 Tag eine verlobte Braut gewesen, hat sie ein Kind. aus der h. Tauff gehoben, Apollonia genannt, Herrn Johann Herpen, eines Vicarii Tochter, so vor der Zeit Pfarrer zum Dendla [Dentlein am Forst, Pfarrdorf im Amtsgericht Feuchtwangen] gewesen. Vier Wochen zuvor, ehe sie mit mir genesen, einen Zahn lassen ausbrechen.

Ist mit meinem Vatter selig 1. zu Weidelbach, 2. zu Dinkelsbühel, letzlich zu Markbrait wohnhafft gewesen, wie oben nach einander zu sehen.

Hat viel Creutz und Ungemach mit meinem kranken Vatter, mit unerzogenen Kindern, mit groben Leuten, mit langwierigen Wittwenstand, so fast 21 Jahr gewährt (dann Anno 1589 26. Jan. gieng er an, 27. Oct. Anno 1609 frühe nach 1 hor höret er auf), ist in ihrem eigenen Hauss, darein sie Anno 1589 12. Maji nach Verlassung des Pfarrhausses gezoger, nachdem sie das Nachtnahl des Herrn vorhin empfangen, sanfit und seelig, als wir hoffen, verschieden, in meinen Beywesen, den 28. Oct. an Simonis und Judae Tag, um 12 Hor, ehrlich begraben, in die Kirch getragen, vom Herrn Pfarrer Georg Conradi mit einer Leichpredigt aus dem 6S. Psalm, von den Wittiben, verabscheidet worden.

B1. 8', fol. 15] Mein des Schreibers dieses Buchs Leben.

Ich, im Titel dieses Geschichtbuchs Benannter, bin gebohren in der heiligen Röm. Reichssladt Dinkelsbühel in einem gemieteten dess Lojens genannten Hauss (dann die Kirchendiener daselbst nicht gewisse Wohnhäuser haben) um eilff hor in der Nacht 7. Januar, Anno 1572 an einem Montag.

Aus einem Calender, Rosae meines Behaltens, hab ich nachfolgende zween Tage mit Willen abgeschrieben:

Anno 1572 7. Jan. 9. Consta. Wag. 15. 3. d $\sigma^{7}$ Schnee oder Wind. 8. Jan. Erhard. Wag. 28. Das letzte Viertel o. 8. n.

Annus 1572 war ein Schaltjahr, vom Anfang der Welt 5j34, gulden Zahl 15, Sonnencircul 13, Römerzinsszahl 15.

Den folgenden 8. Januari frü um 7 hor bin ich von Herrn M. Johann Knauern (so geliehener Neuburgischer Pfarrer zu Dinckelsbühel gewesen und anno 75 gestorben) getaufft und durch Herrn Veit Reinhard, einen alten Greisen, so ein Kirchenpfleger. 
(derer 12 sind und die evangelische Kirchendiener annehmen und bestellen) und Gastgeber gewesen von Hall, aus der Tauff gehoben worden, dessen Weib Anna Herrn Doctor Hambergers zu Tübingen Schwester gewesen, hat mir einen Viertels Talers, so $72 \mathrm{dn}$. golten und damalen ein grosses gewesen, eingebunden und Wolffgang nennen lassen.

Mein Vater M. Wolfgangus Ammonius, Mitdiener am Worte Gottes zu mehrbesagtem Dinkelsbühel, ist aber vom 3. Blat an, wie auch meine Mutter Maria, eine Jungin, vom XI. Blat an gnugsam beschrieben. Meine Mutter hat eine erschreckliche Krankheit, da sie mit mir im Kindbett gelegen, ausgestanden und ist zu End des Maji anno 72 mit der rothen Ruhr, die alten und jungen damahls tödlich gewesen, (NB. inseratur hic si libet) behafftet gewesen. Darum mich mein Vatter abnehmen lassen und gen Feuchtwangen zu der Anfrau gethan, allda ich die rothe Ruhr auch im Anfang des Junii bekommen, von meiner Mutter wieder geholet auf dem Carren, wie mir die Eltern gesagt, wieder angeleget worden bin und gesäuget. In dieser Stadt bin ich in der Kindheit, da ich kaum recht reden kōnnen; es hat sich aber mit meinen Reden biss in 3 Jahr oder drüber verzogen, darumen etliche gesagt und gerathen, die Wehemutter habe mir die Zunge nicht recht gelōset, man muss mirs aufs neue lōsen und aufreissen, der Medicus Doctor Pröbstel aber (so mich fast für ein monstrum naturae und Wundergeburt ausgeben) hat gerathen, man soll mir einen Fisch fürstellen und mich denselben angreiffen heissen, so werds geschehen, wann derselbe schnappe und plezschere, ich etwas herausstossen werde, inmassen auch also gangen, und ich Isch oder Fisch erstlich soll gesagt haben.

Als ich nun, wie gesagt, kaum recht reden können, bin ich in einem kleinen Kinderröcklein zu einen deutschen evangel. Schulmeister Jodoco Gablern in die Schul, weil man keinen reinen evangelischen lateinischen gehabt, gangen, darauf in die (fol. 17) lateinische papstische zu M. Johann Gerlach Rector neben des Pfarrers Knauers Sohn Tobia eine Zeit lang gethan, doch dass ich nicht mit den andern zur Mess und in ihre Kirchen gienge, zum Tagampt. Weilen ich aber einmal oder etlich als ein Kind, so die Sache nicht verstanden, mit andern meines gleichen Ge- 
sellen und Schülern mit zur Mess gangen oder Kerzen getragen und mit Wecken darzu gelocket worden, damit nun die Abgötterey und gleissnerische Ceremonien, so mir wohlgefielen, mich nicht bethörten, hat mich mein lieber Vatter (deme ichs all mein Leben lang Danck weiss) gen Feuchtwang zu meiner Anfrau in die Kost gethan, da bin ich unter M. Johann Hartmann Sommer Rectore in die [Bl. 9] Schul gangen und hab da latein und decliniren gelernet, Vetter Paul Jung hat zu Hauss auch Fleiss gethan bey mir. Ich bin auch darum gar zeitlich gen Feuchtwangen gethun oder geflöhet worden, weil zu Dinckelsbühl pestis grassirt, meine Gesellen und nechste Nachbarn, Bauernfein d genenn, daran gestorben, und mit den Trãgern, wo mir nur der Lufft werden können, ich Sprach gehalten, und wie viel täglich gestorben, wissen wollen.

Anno 1579 10. Juli. bin ich mit meinen lieben Eltern nach Markbrait kommen, folgends da in die Schule gangen und in diesem Jahr schreiben, auch recht decliniren und coniugiren gelernet. Meine Herrn praeceptores sind in diesem Markt gewesen: Herr Johannes Remlein, so hernach ein Rathsherr worden. Philippus Köberer, so 1. Cantor, hernach praeceptor ward, ein gelehrt Männlein und guter graecus, von dem ich viel gelernet; sein Wittib, nachdem er peste geblieben, hat Herr Remlein genommen. Georg Zizmann, von dem ich singen gelernet. Er ist uff meines Vaters sel. promotion als ein Landsmann hieher kommen, letzlich Schulmeister zu Segnitz worden und da gestorben. Nicolaus Agricola oder Bauer Cantor von Helpurg. Der ist hernach Pfarrer zu Krautostheim [B. A. Scheinfeld] und Herbolzheim [B. A. Uffenheim] worden. Bartholomãus Rőder, Cantor und hernach Schulmeister, hat mich auch in musicis dapfer gebraucht, wie ich dann oft unter ihm in frembde Dörffer neben andern Discantisten uff vornehmer Leuthe Bitten gehohlet worden zu Hochzeiten. Herr Joh. Röschius Praeceptor, der mich in versibus endlich geübt.

[f. 18.] Anno 1586 am Tag Martini gen Rotenburg $m$ it meinen Vatter selig uff der Gutschen gefahren, der mich zu Herrn Johann Suevo oder Schwaben praeceptore in der III. Class in die Cost verstellet, als zu seinen Landsmann, das Jahr um $32 \mathrm{fl}$. Die andere 5, so lauter Edele und patricii waren (unter welchen auch die zwene Archiv für Kulturgeschichte. I. 
Neurhat, deren einer Amtmann zum Seehauss [B. A. Scheinfeld] worden) haben ie $36 \mathrm{fl}$. geben; bin aber in dieser Cost nur biss auf den 23. May anno 87 blieben aus gewissen Ursachen. Diese Nacht hat mir fast ganz durch mein Vater seliger fürgeprediget, zum guten ermahnet, vom bösen abgewarnet, folgenden Tags den Rectorem scholae zu Gast geladen und mich ihme befohlen, darauf hat es nasse Augen und Abschied gesezt.

Anno 1587 23. May bin ich daselbst bey Hanss Geissendörffer, Nadlern und Cramern, in der Hafengassen angestanden, das Jahr um $27 \mathrm{fl}$. und $1 \mathrm{fl}$. Waschgeld, da hab ichs besser gehabt.

In dieser Stadt Rotenburg hab ich zu Praeceptoren gehabt in secunda Classe

1. Herm Simon Hornung, der ein Crămer darneben, hat mich sehr lieb gehabt und geweissaget, ich werd ein Hoffprediger mit der Zeit werden; hat auch, da er nach Brait verrücket und die Schwäbin (so hernach von $\mathrm{lhm}$ die Hornungin genannt) genommen, gesagt, weil ich zum Seehauss so viel geprediget meinem gn. Herrn, da sey die Weissagung erfüllet. Ist dieser Herr zu Markbrait ein Rathherr gutes Vermögens gewesen, hat Herr Wunderleins Haus gebauet und ist daselbst entschlaffen.

2. In prima Classe, darein ich anno 876 . Jun. transferiret und gesezet, ward Herr M. Johannes Schemel mein Rector und günstiger Förderer, der hat [Bl. 9'] mich in graecis wohl geübet, da ich grichische Scripta gemacht. Denn ich Demosthenis orationes Olympiacas neben dem Evangelio graeco gehört.

M. Melchior Neander, Conrector, der hat mich in poesi fein informiert, da auch Herr Minderlein, unter dem ich sonst nicht gesessen, viel dabey gethan, wenn ich privatim in der Class für mich selbsten an der Tafel laboriret nach der Schul.

Sie haben eine venam poeticam an mir gesagt seyn. [fol. 19.] In dieser Stadt hab ich anno 87 in Weihnachten uff einwilligen meines Vatters um mehrerer Übung willen mit den armen Schülern und alumnis umgesungen und $1 \mathrm{fl}$. bekommen, bin aber, ob wir wol vielmal eingelassen und im Spital gut Sach gehabt, hefftig erfroren, und weil ich bey einen Schüler gelegen, kräzig worden und viel Jahr nit wol zurecht kommen können. 
Ich hab auch in dieser Stadt eine Comoediam latinam Frischlini, Rebeccam, helffen agiren und, weil ich etwas leibig, gastrodis person vertretten.

Als ich aber, weil nur 4 Meil heim waren, einsten heimkommen und mich etliche Wochen zu Hauss uffgehalten, und weilen uffs Examen alles hernach zu lernen mir zu viel w'erden wolte, besonders in der grossen grichischen Cousii grammatica, bin ich der Schulen Rotenburg nit gut worden. Darauf hat mich mein Vatter seliger, weil ichs in der Nähe nicht erleiden können, in die Ferne gethan, mit Herrn Secretarii Horns und seiner Commendation gen Hoff ins Voitland geschickt und uff die arme Schul daselbst, mein Brod zu ersingen lassen gemeint. Aber der Herr Rector, nachdem er aus dem Schreiben und meinem Bericht vernommen, ich von gnädiger Herrschafft Seinssheim jährlich eine Beyhülff von $15 \mathrm{fl}$. hette, hat mir eine Cost darumben ausgangen bey einem alten Büttner, Claus Zeitler genannt. So bin ich nun den 10. Augusti am Tag Laurentii gen Hoff kommen, da eben die Mess waren, und Herr Marggraff Georg Friederich von Brandenburg samt dem Herrn Administratore zu Hall mit einer grossen Anzal Raisigen in der Stadt lagen. $\mathrm{Da}$ ist man $8 \mathrm{Tag}$ (wie in Messen man pflegt) nicht in die Schul gangen.

Anno 1588 den 19. Aug. bin ich in die erste Class versezet worden und hab allda zu Herrn Praeceptoribus gehabt, wie im folgenden Blat zu sehen.

[fol. 20.] Der Rector war M. Christophorus Cadesreuter senior, an den ich verschrieben von meinem Vater seelig und Herrn Secretario Johann Horn, dessen ich wohl genossen, und ist er Herr Rector mit mir persönlich ausgangen, in der Stadt eine Cost mir auszugehen und zu wegen zu bringen, so lang biss er einen alten Büttner, Zeitler genannt, angetroffen und mich dahin bracht. Dieser selige Rector ist anno 1589 6. October verschieden.

M. Thomas Blebelius Conrector hat hernach dem vorigen succedirt im Rectorat, mich in hebraicis und astronomicis, auch rhetoricis unterwiesen, da er auch von allen der (!) unterschiedliche Bücher geschrieben (wie jener $M$. Cadesreuter seine graecam grammaticam tradirt), auch mich an meine gnädige Herrn von 
Seinsheim recommendirt, neben Herrn Doctore Theologiae Aurelio Streitbergern. ')

[BI. 10, f. 135] Omnibus lecturis has literas cum debita unicuique honoris mentione s. d.

Cum ipsa per se testimonia magni facienda esse arbitramur a viris literatis et iis, qui de ingeniis discentium literas et linguas Ecclesiae Dei et communi vitae necessarias iudicare porsunt profecta: tum quia ad persequendas laudes studioso homine dignas, bonae indolis naturae his testificationibus excitantur; est etiam bonae conscientiae signum, praeceptorum de se existimationem non extimescere et verecundiae argumentum Maiorum iudicio se reverenter subiicere. Haque et hactenus testimonia concessimus scholae nostrae Curianae alumnis, pietatem in vera Dei invocatione, modestiam in regendis moribus, diligentiam in urgendis literarum studiis et obedientiam suam erga praeceptores nobis approbantibus. Et nunc praesenti honesto adolescenti Wolfgango Ammonio Zeapolitano [Zea, der Dinkel], reverendi et doctissimi viri domini M. Wolfgangi Ammonii, eius loci pastoris pie defuncti filio, elogium damus pietatis, observantiae erga omnes et in primarum artium et linguarum studiis assiduitatis. His enim nominibus hoc fere quadriennio, quo in schola nostra Curiana cum laude modestiae et diligentiae versatus est, se nobis omnibus approbavit, Praeceptoribus et quibus cum vixit hospitibus carus et gratus fuit, spem etiam de se non vulgarem concitavit. Cum igitur existimemus, eum aliquando (praecipue si studiorum curriculum feliciter inceptum oìy $\vartheta \varepsilon \bar{\omega}$ urgere perrexerit) patriae suae utilem et necessarium civem futurum, diligenter ipsum commendamus omnibus bonis et doctis viris, quibus Ecclesiae Dei salus et res literaria curae est; in primis vero illustri et generoso Domino Georgio Ludovico a Seinsheim in Hohencottenheim, Seehaus et Sinchingen libero Baroni, studiorum patrono et fautori optimo, domino nostro, omni reverentiae cultu dignissimo: cuius generositatem reverenter et summisse oramus, ut sua liberalitate et stipendii beneficio huius Wolfgangi subditi et clientis sui pupilli egestatem summam sublevet, ne feliciter incepta studia propter inopiam et paupertatem abiicere

1) Vgl. Beitr. 2, 134 Anm. 1. Aurelius Streitberger war 1577 Inspektor des Gymnasiums $2 u$ Hof und starb 1612, 69 Jahre alt. 
cogatur, sed vestrae [fol. 130] generositatis munificentia et auxilio his ad prooptatam metam Deo iturante perductis aliquando ecclesiis alt scholis utiliter inservire queat. Hoc enim beneficio impetrato in nos recipimus ipsum, uti probum Beneficiarium decebit, vitam cum pietate, honestate, debita in studiis gravioribus diligentia et animi gratitudine coniunctam imposterum etiam acturum et summis viribus operam daturum, ne eiusmodi liberalitatis beneficium et sumtus in ipsum frustra collocentur.

Hoc et Deo gratum et Ecclesiae utile erit et hic Wolfigangus discipulus noster vicissim sedulitate in studiis, pia precatione pro communi salute et omnibus obsequiis officiis debitam animi gratitudinem vestrae generositati toto vitae suae tempore declarabit. Et nos quoque hanc effusam liberalitatem et iuvandi studium apud omnes celebrare studebimus. Bene in Christo vale, illustris et generose domine Baro a Seinsheim cum pio et benevolo Lectore et quos semper favore, benignitate et liberalitate cum autoritate coniuncta literas earumque cultores prosecuti estis, fovete et defendite. [Bl. 10'.]

Dat. Curiae Variscorum ad ripam Salae fluminis 12 Calendas Maji Anno gratiae MDXCII.

Aurelius Streitbergerus, s. theol. D., Superintendens scripsit.

M. Thom. Blebelius Budissinus, scholae Cur. Rector et suo et collegorum suorum nomine scripsit.

[fol. 20] M. Enoch Widmann [Enoch Widmann's Chronicon Curiae in Menckes Scriptores Germaniae, Lips. 1730, Tom. III, 756. Deutsch in Hohenzollersche Forschungen 1893 S. 244], der hat mir in Poesi graeca et latina wohl gedienet und weil er mich zu der Löwin in die Kost verstellet, allda er ein Vetter gewesen, privatim mir viel untersagt und gewiesen, ist nach meinem $\mathrm{Ab}$ schied Rector worden.

M. Simson Moncelius hat mir auch in lateinischen versen wohl geholffen und ist ein getreuer Praeceptor gewesen, so Anno 88 23. Decembr. ins ministerium kommen uff dem Land, hernach offt und viel promovirt worden, ist leztlich Prediger zu Heilsbronn und Dechant zu Neustadt an der Aisch worden.

M. Georg Thecius oder Deg hat mich sehr lieb gehabt, in grichischen poeten und soluta oratione mir wol zugeschlagen. 
Weinet bitterlich 23. Jun. anno 92, da ich ihn valediciret und gesegnet; soll ein Rathsherr worden seyn.

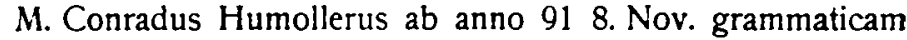
latinam tractirt, sonderlich doctrinam de accentibus uns fein geweiset.

So bin ich nun in dieser Stadt Hof fast 4 Jahr gewesen und hab da meine beste Sachen gehabt bey obgenannter Barbara Löwin, die eine Cramerin gewesen und einen grossen Sohn Hannsen gehabt, so ihr die Haushaltung und Cram versehen. Den kleinern Sohn Philippum hab ich privatim singen und anders gelehret 3 Jahr.

[fol. 21] Hab ihr, dieser Costfrauen (nachdem ich bey dem Büttner Zeitler nur $1 / 4$ Jahr geblieben, um des argen Weibes willen, so nitgern essen sahe) das erste Jahr $15 \mathrm{fl}$. [gegeben, das andere aber 12 fl. und noch folgende $2 \mathrm{Jahr}$ dergleichen. Doch hab ich darneben arbeiten und schreiben müssen, was man mich geheissen, und gleich wohl meine Ergőzlichkeiten darneben gehabt und allen guten Willen verspühret.

Anno 90 im Majo, weilen die Primani fast alle im Brett spielen gekönnt, es auch gelernt. Item Anno eodem 27. Maji uffn Rücken schwimmen mit den Meelführer, so leztlich Abbt zu Heilsbronn worden.

Anno 901 . April uff der Cithern lernen schlagen.

Anno 1591 8. Jan. hab ich angefangen graeca carmina zu schreiben, wie im vorigen 90. Jahr 6. Nov. Phalaecia und 3. Dec. Sapphica Carmina Latina.

Anno 91 22. Jul. das höfische Stat-Chronicon, so M. Widmann [Enoch Widmans Chronik der Stadt Hof. Nach der Originalhandschrift zum erstenmal herausgegeben von Christian Meyer 1893] verfertiget, neben andern (darunter auch Melfürer war) alle Tag 1 Stund nach der Abendschul helffen abschreiben selbfünfft. Welches anno 92 den 23. Martii fertig worden, darau: sind wir von ihme zu Gast geladen und herrlich tractiret worden.

Anno 91 16. Sept. den Buchdruck Pfeilschmids ${ }^{1}$ ) gesehen und sobalden in mein Stammbuch etwas lassen drucken, wie auch den 29. Dec. in Herbsts Stammbuch. [B1. 11]

1) [Beitr. 4, 65 Anm. 5] Matthäus Pfeilschmidt, 'Buchdrucker in Hof, schrieb 100t ein Gesangbuch. 
Anno 92 31. Mart. angefangen, Partes zu schreiben, welches mir hernach zu meinem Cantorat wohl gedienet.

Eh ich vom Hof weg gezogen, hab ich einem Landsmann Arnoldo auch ein Buch aus dem Virgilio fürgelesen und expliciret. Anno Domini 1592 am Tag Petri Pauli bin ich früh Morgens nach Jena kommen und hab noch diesen Tag die Bacchantenhörner lassen abwerffen und dagegen einen studiosum im Wirthshauss bey der gulden Gans angeleget. Doctor unius Juris Jacob Welser war mein absolvens. Paulus Lucius ward auch mit mir deponirt. Johann Hohndorff, so hernach ein Minister Ecclesiae worden, war Depositor, und ich bin der 1295., so er gehefelt; meine Zeugen waren Johannes Herbst (der hernach in Doctorem Juris promovirt) und Johann Cobmen [fol. 22]; costet mich dieser actus 2 fl. 4 groschen 4 dl. Anno 92 1. Jul. bin ich von Herrn Doctore Nicolao Reusnero, dem hochberühmten Juristen, der viel Bücher geschrieben und dissmal Rector Magnificus der Universitaet Jena war, ins Studentenbuch immatriculirt und einverleibet, costet 6 groschen. Folgende Tage habe ich meine commendationes ausgetragen, so mir Herr Matthaeus Herbst, Pfarrer zu Erlach [im Amtsgericht Ochsenfurt], an Herrn Doctor Müllern, Theologum, Doctor Johannem Schrötern den āltern, den perpetuum Academiae Rectorem, und M.Hammern, der Hebraischen Sprachen professorem, ertheilet hatte. Welches ich dann würklich genossen und dem $M$. Hammern sonderlich lieb gewesen bin und viel gutes von ihm studiret. Er hat mich auch mit ihm zu seinen guten Freunden und Verwandten umher ausser Jena geführet, zu Apolla und anderstwo; da unter andern ein Pfarrer, als er meinen Zunahmen gehöret, gesagt: magnum nomen, magnus vir, uff ein grossen Nahmen gehör ein dapferer Mann.

3. Aug. die 1. lection gehört von $M$. Haidero und D. Reudenio.

Uff dieser hohen Schul Jena hab ich zu Praeceptoribus gehabt und gehört in Theologia Doctor Georg Müller, von dem ich die augspurgische Confession hören expliciren und epistel ad Romanos. NB. Er fieng den 9. Jul. 1594 an, auswendig zu profitiren, und dictirt nichts.

Doctor Ambros Reudemium, der gar fidelis war, etliche 
Psalmen explicirt (114 ist mein anfang) und Isagogen biblicam gelesen, auch seine privata collegia gehalten, da ich auch bey. Doctor Samuel Fischern, so Superintendens, und Johannem gelesen.

II. In juridica facultate, welche ich erstlich studiren sollen (vermög habenden schrifftlichen Befehls) Doctor Liberium Hoffmann gehört in regulis Juris; item Doctor unius Juris Jacob Welsen (so den Ranum [Pierre la Ramee $† 1571$ ] selbst gehört hat zu Paris) über die 2 erste Bücher in institutionibus juris, daran 5. Octobris anno 92 angefangen; in hebraica, chaldaica et syriaca lingua $M$. Christoph. Hammerum, sanctae linguae professorem, der auch Joelim und Jonam gelesen. [fol. 23] In graecis hab ich gehört $M$. Laurentium Rhodomannum [ $\dagger 1606$ in Wittenberg], der ein lumen Germaniae gewesen und eine selzame pronunciation gehabt, wie die heutige Grichen, Theogoniam Hesiodi lass er und weist uns frei die imitation. In Mathematicis von $M$. Georgio Lymnaeo die sphaeram, Theorias planetarum, Calendar. Ecclesiasticum, tabulas prutenicas, Arithmetic etc.

In physicis und Libell. de Anima Doctor. Zachariam Brendel, der ein herrlicher, getreuer, gottsfürchtiger Medicus und praeceptor (ist im September anno 26 verschieden).

In Ethicis und Logica den $M$. Wolffgang Haider, der ein lustiger sehr gelehrter Mlann. Von diesem hab ich privatim eine specialem doctrinanı de virtutibus studiret (er ist im 18. Sept. anno 1626 begraben).

In Poesi Eliam Reusnerum Licentiatum gehöret.

Bl. 11'] 23. Sept. 1592 die erste disputation zu Jena, aber den 28. Nov. anno 1588 zum Hoff gehöret. 28. Octob. 1592 ich das erste mal disputirt.

Mein erster Stubengesell war Johann Herbst (mein Landsmann und höfischer Schulgesell) vom 29. Jun. Anno 92 an bei M. Lorenz Evo oder Frūeauf, der professor Arithmetices vor diesem gewesen und schier gar erblindt. Hernach 22. Sept. sind wir zu Nicol. Müller, Becker in der Leutergassen, gezogen, das Jahr um $6 \frac{1}{2}$ fl. Stubenzins. Mein anderer Stubengesell und allerbesten Freund einer (dann den Christoph Jordan, so mit der Zei! 
Superintend. zum Hof worden, halt ich für den besten) war Johannes Dressel von Neustat an der Hayd [südlich von Sonneberg], 2 Meil ron Coburg gelegen, der hat mich Bett und Stuben frey gehalten, ich ihn dagegen privatim unterrichtet an gesagten Ort. Vom 30. April Anno 94 aber haben wir in der Bildschnizerin Hauss, im engen Ciässlein gewohnet.

NB. anno 1593 hab ich rom XI. Febr. an Logicam Rami geiesen.

Weil ich nun auf der hohen Schul nit lang zu bleiben gelabt, als hab ich mich bald uff eine facultaet begeben müssen und demnach gnädiger Herrschafft Sainsheim Herrn Georg Ludwig von Sainsheim [der jüngere 1554-1599] heimgestellet, die mir erstlich das Studium Juris gnädig anbefehlen lassen, wie oben auch gedacht, ich soll mich aber mit Büchern [fol. 24] und Kleidern nit überlegen und heut oder morgen nit abkaufen, sondern meinem Herrn verbunden seyn und bleiben. Nach etlichen Wochen ist ein anderer Bescheid kommen, als wenn Ihre Gnaden nichts von vorigen Schreiben wüsten, ich soll aber zu meinen Freunden mich herausbegeben und von ihnen Bürgschafft zum studio Juris ausbringen oder das Studium Theologiae ohne Bürgschafft fürnehmen. Als ich nun anno 93 den 13. und 14. Jul. ihres Raths zu Feuchtwangen gelebet, rhieten sie einmüthig, was mein Vatter studiret hätte, da solit ich auch mich beyfinden lassen. Dem hab ich auch gefolget und dank Ciott dafür.

Den 15. Jul. nechsten Tags hinnach dem Rath hab ich zu Dorf Gütingen uff meiner Mutter Bruders als Pfarrern Erlaubniss frül morgens die epistel 1. Pet. 3 gelesen in der Kirchen, neben einem capitel aus Lucae 4 und die collecten gesungen, abends aus der Kinderpredig das dritte Gebot gelesen und ist diss gleichsam mein erste prob in Kirchen actibus.

Als ich nun wieder nach Jena kommen, hab ich den 26 . Augusti anno 1593 (war der XI. Sonntag nach Trinitatis) meine erste Predigt (welche ich voriges Tags 25. Aug. in eines armen freuleins Haus vor meinen Stubengesellen und guten Freunden privatim gethan) offentlich zu Zwaitzen [Zwätzen], so eine halbe Meil von Jena gelegen, in der Kirchen gehalten, in Beyseyn Herrn Pfarrers und 5 Studenten. Da hat mir nun Gołt meine Bitt gewärt, und 
ich ein Gelübd gethan, welches ich gehalten. Ist auch also gangen, wie ich in meines Frãuleins seligen zu Elsa Garten besonnen.

9. Sept. anno 93 13. Trinit. hab ich zu Lichtenhan [Lichternhayn], 30. diss., 16. Trin. zu Amerbach [Ammerbach] geprediget.

9. Decemb. 2 Predigt gethan, 1) zu 14 Heiligen [7 Kilometer von Jena, gegen Apolda], ist ein Dorff also genannt, 2) zu Rumstat, und sind ich und mein gut Freund Christoph Kom, weils eine grosse Meil Wegs, mit der Latern in der Nacht biss Tag gangen, in grossen Ungestüm.

[fol. 25] 25. Decemb. Anno 93 zu Liechtenhan wiederum.

26. Decemb. zu Amerbach. 27. Decemb. abermal zu Liechtenhan in Beyseyn M. Melzers, Jenischen Caplans und der Seinen gepredigt, hab auch ein statliche Malzeit uff des heligen Casten mit ihnen besessen.

Anno 1594 27. Jan. zu Liechtenhan abermals gepredigt. 10. Febr. zu 14 Heiligen. 24. Febr. zu Liechtenhan. 10. Mart. zu Ziegenhan. 24. diss zu Ziegenhan und Prissniz [Jenaprisnitz].

[Bl. 12] 31. diss 3 Predigten an einem Tag gethan, 1) zu Jeschwiz, 2) zu 14 Heiligen, 3) zu Klein Rumstat.

1. April zu Grossen und Kleinen Rumstat geprediget, auch ein Malzeit zu Grossen Rumstatt uffs Fest genossen.

14. diss zu Jeschwitz mich exerciret. 9. May wieder daselbst. 28. Jul. zu 14 Heiligen und Rumstat geprediget. 20. Octob.zu Amerbach.

Ja ich hab etliche Exercitia nicht uffgeschrieben, dann ihrer bey 30 gewesen. Unter andern aber, so mir wiedrig zugestanden in Jena, ist nicht der wenigsten eins, dass ich gegen meinem gnädige Herrn bin versagt und angossen worden; hab demnach 5 Testimonia und Zeugniss als Herrn D. Müllers, D. Brendels, M. Hammers, M. Lymnaei und M. Melzers gen Seehauss geschickt und der falschen Anklag damit gesteuert.

Wolfgangum Ammonium quamvis consuetudo frequens hactenus non familiarum mihi tradidit: Praeceptorum tamen testimonia ita commendant et annotata ex lectionum mearum auscultationibus consignata, ita officio functum esse probant, ut tempus $a b$ illo minime inutiliter collocatum esse iudicem: Quod ego rogatu ipsius, meo hoc autographo testari non sum gravatus. Scripsi 16. Febr. 1594. Jenae. 
Georg Mylius D. et Professor Theologus.

Illustris et generose Baro, Wolfgangus Ammonius, generositatis Tuae Alumnus, studiorum suorum testimonium a me petiit. Quia vero hic ante biennium ferme a viro reverendo domino Matthaeo Oporino, condiscipulo meo veteri, mihi commendatus fuit, operam dedi sedulo, ipsius ut et vitam et studia observarem et si quae corrigenda forent, libere corrigerem. Quare, cum talem deprehenderim, cui cordi et curae literarum optimarum studia sint, non modo Joebeae praelectioni Auditorem sese mihi quoque praebeat, non potui in honesta ipsius petitione hac non acquiescere. Eum ergo cum reliquis Reverendis et Clarissimis Viris Collegis meis de nota meliori generositati tuae commendatum esse percupio submisse petens, ut imposterum quoque ipsius studia p. A. provehere non dedignetur, quod Deo et generositati tuae atque Ecclesiae patris sui, viri doctissimi, p. m. instar commode inservire aliquando possit, non dubito, quin daturus sit operam quoque, quo animi grati significationem generositati tuae vicissim effuse probet. Vale in Christo generose Baro, et huius (gehöret vielleicht noch hinzu Academiae) [fol. 138] discipulos atque Ministros, ut hactenus laudabiliter et liberaliter fecisti, ita in posterum etiam Tibi commendatos esse patere. Jenae 16. Febr. anno Christi 1594.

Generositalem tuam submisse colens $M$. Christophorus Hammerus Linguae sanctae professor.

Dominum Wolfgangum Ammonium operam mihi dedisse sedulam publice docenti physicen et librum de anima testor Zacharias Brendel Med. Doctor, Professor publicus in Salona. Actum Jenae 16. Febr. 1594. Zacharias Brendel D. S. I. C.

B1. 12'] Petiit a me ornatissimus Juvenis Wolfgangus Ammonius studiorum suorum testimonium, quod Patronis suis exhiberet. Cum autem aequum non sit commendationes denegari is, qui merentur, libenter obsecutus sum huic petitioni, et quae comperta haberem, perscripsi. Testor igitur nominatum Ammonium singulari diligentia hactenus mathematicis disciplinis, quarum professio ab illustrissimis Saxoniae Principibus, huius nostrae Salonae Nutricis Dominis nostris clementissimis mihi demandata est, operan dedisse, ut facile coniiciam reliqua studia non minus ipsi curae fuisse, cum diligentius gravioribus insudarit, spemque concipiam utile eum 
futurum organon Ecclesiae et scholae. Ideoque debita animi sub)missione a generosis Nobilissimisque huius studiosi Patronis peto, ut sua gratia et ope ulterius ipsi adesse non graventur. Erit hoic, ut speramus et ex animo precamur, Ecclesiae Christi tam utille quam quod utilissimum. 16. Febr. anni 1594 Jenae.

Georgius Lymnaeus $M$.

NB. quintum testimonium deficit.

[fol. 20.] In diesem 94. Jahr wär ich gern Magister worden und hab alles, was darzu gehöret, in Bereitschafft gehabt, allein an Geld fehlete es, muss demnach aus Armuth und weil mir Niemand darzu helffen wolte, mein Vorsaz āndern.

10. Maji diss Jahrs bin ich in H. Doctoris Reudemii Theologicum collegium kommen.

Anno Christi 1595 14. Mart. bin ich von Jena abgefordert worden, weil der Herr Herbst, Pfarrer zu Erlach, gestorben, ihme zu succediren (wie es erstlich gemeinet war) oder aber im Carntorat an Pfisters stat zu kommen.

Bin am Sontag Laetare oder Mitfasten zu Brait ankommen.

In der Charwochen und Ostertagen hab ich die Predigten zu Eilach und Sundheim [Kaltensondheim bei Kitzingen] uff gnādigen Befehl verrichtet anstat des von Ampferach [im B. A. Feuchtwangen] herkommenden Herrn Schwagers Schechsii, welchen Johan Pfister mein Antecessor succediret hat. Darauf die Schul zu Markbrait an Herrn Pfisters stat, der nach Ampferach zum Pfarr erhoben worden, versehen uff gn. Begern der Herrschafft vom 25. April an, biss Anno 1595 10. May mein gn. Herr Herr Georg Ludwig von Sainsheim in der Person mich in Beyseyn Herrn Schuldheissen Nicolai Grohen und aller seiner Geistlichen zum Braiter Cantorat eingeweiset und investiert.

Den 13. May darauf ins Cantors Heusslein eingezogen, da hernach die Kirchner wohnen.

In diesem 95. Jahr hab ich geheiratet, und mein.1. Verlöbniss in Beyseyn meiner Mutter 2 Brüdern Herrn Abraham Jungen, Pfarrers zu Dorf Gütingen, und Herrn Philipp Jungen, Pfarrers zu Leutershausen, am 9. Octobr. gegen Abend gehalten, [fol. 27] mit Apollonia Kuppelichen, da ihre Mutter auch dabey und ihre 2 Brüder Jacob Kuppelich, ein Vogt zu Feuchtwang, 
und Georg Cuppelich, ein Beck daselbst, samt ihren beyden Weibern und dem ledigen Bruder Abraham; ist alles verrichtet worden im Dorff Gütinger Pfarrhaus.

Geschlecht und Herkommen meiner lieben Apollonien.

Ihr Vatter, mein lieber Herr Schweher seliger, hat geheissen Johann Kuppelich, ist zu Feuchtwangen bürtig anno 1527; sein Vatter, meines Weibs Anherr, hat auch Johann Kuppelich geheisen und ist ein Schuster gewesen, hat aber das Handwerck nicht getrieben, sondern die Wirthschafft auf den Marckt in dem Hauss, so iezt Schwager Friedrich Alexand. Kuppelich besizet, und er durch Heirat bekommen, ist zue Hardt [Haardt] bey Weissenburg daheim gewesen.

Dieser mein gesagter Schweher hat das Beckenhandwerck gelernet und eine [Bl. 13] Zeit lang getrieben, ist aber ein Rathsherr dabey gewesen, hat lezlich das Statvogtampt um seines Wohlverhaltens willen bekommen und das Handwerk aufgeben. Sein erstes Weib hat Magdalena geheissen, eine Tochter N. Alberts, und anno 1547 2. May mit Ihr Hochzeit gemacht, in die 18 Jahr in der Ehe mit ihr gelebet und 10 Kinder mit ihr erzeuget, unter welchen eine todte Leibesfrucht gewesen. Seine erste Tochter ist gewesen Margaretha, so zu Georg Funken, einen Bauern in Wissath (Wieseth bei Feuchtwangen) geheurathet, ist ehe verschieden, denn der Vatter, ungefehrlich Anno 1583.

Das andere Kind Johannes anno 1549 23. Aug. geboren, ist zu Crailsheim an der Pest gestorben anno 1613, der ist ein Müller und Rathsherr zu besagtem Crailsheim und sehr berühmter Mann; hat mir viel guter Rāth ertheilet, durch seine Rechtshändel aber in grosse Ungelegenheit kommen, doch sehr gottseelig blieben. [fol. 28.]

Das dritte Kind hat Laurentius geheisen, so anno 1552 26. December geborn, hat fein studiret, ist ein mächtiger Historicus gewesen, also dass er sich berühmen kōnnen, es wer kein Geschichtschreiber zu seiner Zeit herauskommen, den er nicht in seiner Liberey hätte. Ist erstlich infimus scholae Feuchtwangensis Collega worden, hernacher Vogt zu Wassertrühdingen, ein reicher ansehnlicher Mann, ist daselbsten verschieden den 17. Jan. anno 1620.

Das vierte Kind Georgius, so ein Beck worden zu Feuchtwangen, ward gebohren anno 155424 . December, ist auf seiner 
Schwester Amaley Hochzeit anno 1598 7. Nov. von seinen Müller zu Feuchtwangen, der Schön Müller genannt, [confer fol. 158] dà die andern getanzet, erstochen worden, doch ehrlich begraben; ich hab ihm vorgebett in Todesnöthen. Desselben Müllers Sohn, ein Studiosus, ist von einer Tanzbodenstiegen herab zu Tod wiederum gefallen.

Das fünfte, Maria, geborn anno 1557 20. Jan., ist zu Tieffenbach [B. A. Hilpoltstein], als ich diess geschrieben, meines Wissens, noch am Leben.

Das sechste Kind Jacob ist geborn 155827 . Octobr., ist zu Feuchtwangen Vogt gewesen, im Jahr 1600 22. Novemb. verschieden, lang wohl discipliniret zuvor.

Das VII. Ottilia, so meinen Vettern Herrn Abraham Jungen, Pfarrern zu Dorff Gütingen, gehabt, eine lange Zeit, ist anno 1561 16. October gebohrn.

Das VIIl. Abrahamus I. anno 1563 28. Jan. geborn, den 2. Febr. darauf verschieden.

Das IX. Magdalena, die Heinzen Müllerin genannt, ward geborn anno 1564 29. Junii, verschied ums Jahr 1590.

[f. 29.] Anno Christi 1565 16. Octobr. am S. Gallintag hat mein 1. Herr Schweher das andere Mal Hochzeit gehalten mit meiner lieben Schwiegermutter Apollonia (so ein Tochter Georg Jungens gewesen), mit welcher er auch durch Gottes Segen 10 Kinder erzeuget, deren das erste und andere im Kindhaben gestorben (sie aber die Schwieger selig selbsten ist anno 1609 11. Jan. im Dorff Aw (NB. in der Landkarten heissts Ah) [Aha] nit weit von Gunzenhausen bey ihrem Sohn, Herrn Abraham, Pfarrern, an der Wassersucht blieben.

Ihr drittes Kind war eine Tochter Apollonia prima, geborn anno 156822 . Julii, hat nur 31 Wochen 1 Tag 12 Stund gelebt.

Das IV. Ihr Kind ist Abraham Kuppelich anno 1570, 14. Ocober geboren, am Tag Calixti, der hat nun, als er zu Jahren kommen, wohl studirt und ist Pfarrer im Fürstenthum Brandenburg worden zu besagten $A w$, auch hernach eine lange Zeit Senior im Capitel Gunzenhausen von anno 12, wie ich im Augusto erfahren, auch Pfarrer zu Samenheim [im Bezirksamt Gunzenhausen], dero Zeit noch am Leben, hat Hochzeit gehaiten bald nach uns. 
Das V. Apollonia secunda, meine liebe Haussfrau, nunmehr selig, diese war geborn anno 1572 5. May Montag zwischen 10 und 11 Uhr in der Nacht; ihr Tod stehet unten.

Das VI. Kind Catharina ward anno 1574 27. Mart. geboren, hat einen Mezger Melchior Gopelt zu Krailsheim zur Ehe gehabt, mit dem sie anno 1597 12. Jul. Hochzeit gehalten; stirbt am Kindhaben, ohngefehrlich ums Jahr 1618.

Das VII. Kind Barbara hält man dafür, sie sey anno 1576 gebohrn, hat einen Becker zu Gunzenhausen, bey deren Hochzeit wir beyde Eheleuthe gewesen mit grossen Costen.

Bl. 13'; Das VIII. Amaley genannt (so erstlich einen Infimum Scholae zu Feuchtwangen Georg Ziegelmüllern gehabt, der hernach zwo Pfarr noch bezogen Grāfensteinberg [im Amtsgericht Gunzenhausen] und Berolzheim [im Amtsgericht Heidenheim], aber nach seinem Absterben hat sie einen Wirth bekommen), soll ums Jahr 1578 gebohren seyn. Ihr Herr seliger ist verschieden 13. Sept. anno 14, hat mit ihr Hochzeit gemacht Anno 98 7. Nov.

[fol. 30] IX. Kind ist Friderich Alexander, geborn anno 79 Montag nach Palmarum, hat das Becker- und Barbierer-Handwerck gelernet, ist zu Feuchtwangen lang Zeit Umgelder und Wirt gewesen, lebt noch, hat Hochzeit zu Feuchtwang 26. Jan. anno 1602.

$X$. Kind hat Anna geheissen und ist anno 1583 gebohrn, diess Kind hab ich nie zu sehen bekommen.

Mehr besagter mein erster Herr Schweher ist anno 1585 30. Januar. in Gott seeliglich verschieden mit einem statlichen Lob und Betrauernus. Doctor Adam Jung, meiner Mutter Bruder seeliger, hat ihm das Zeugniss gegeben, dass in ganz Feuchtwang, seinem Vatterland, ihm niemand grössere Ehr als er erzeiget habe.

Nun komm ich wieder auf meine erste Ehrnfrölichkeit, die hab ich zu Feuchtwangen gehalten anno Christi 1595 . Uns hat in der Stifftskirchen copulirt M. Lorenz Albrecht, unser Gefreundter, ein anderer Caplan hat die Hochzeitpredigt gehaiten aus dem 1. Buch Mose 2. capitel: Es ist nicht gut, dass der Mensch allein sey. Ist gewesen Diensttags den 18. Novembr.

Unter andern Hochzeitgästen waren Herr Geyer der alte Vogt von Ampferach wegen des wohlgebornen meinen gn. Herrn von Sainsheim Georg Ludwig etc. ein Legatus (so mir 3 Reichs- 
taler verehret). Item Herr Philipp Jung [fol. 31] M., Pfarrer z:u Leutershaussen, ein Bruder meiner Mutter, item Herr Abraharm Jung, Pfarrer zu Dorff Gütingen, auch meiner Mutter Bruder, mit seinem Weib Ottilia und Tochter Evalein, mehr ein anderer Mutter Bruder Paulus Jung, Kornschreiber zu Onolzbach. Item Vetter Herr Hanns Jung studiosus, so hernacher Registrator zu Weickersheim worden, vorhin aber Herr Johann Erkingers von Sainsheim praeceptor war, etc.

Auf der Braut Seiten waren unter andern auch Hochzeitgāste der Herr Dechant Eck, ein ganzer erbarer Rath zu Feuch twang, Herr Lorenz Albrecht, der uns eingesegnet, auch die Frau Amptmannen, eine Edle, wie auch des andern Tags ein Jungkher von Düna, Herr Abraham Cuppelich, ein studiosus, und Friedrich Alex. Cuppelich, ihre rechte Brüder, etc.

[fol. 32] Darauf sind ich und meine Apollonia heimgefahren mit den Unsern und haben unser eigen Hausswesen angefangen. Und, ob mir wohl der Anfang zu meinem ehlichen Leben zimlich, ja sehr sauer ist worden (sintemahl ich biss gen Jhena hin und her gesprenget, uff 48 Meil aus falscher Nachred und Verdacht, deren Gott Lob sich keines gefunden und sonst zu der Freyerey, auch Einladung der Gāst und Ehrenfrölichkeit widerum auf 42 Meil Wegs in Kurzen raisen müssen, ohn was ich gen Seehaus hin und wieder (darzu mir meine vorgesezte Geistliche dapffer die Brände geschüret und doch nichts erweisen können) getanzt. Ob, sag ich noch einmahl, der Anfang meines Ehewesens mir zimlich sauer gemacht worden und ich fast 100 Meilen herum terminiren müssen mit nit geringen Unkosten: jedoch ist meine Unschuld frey am Tag kommen und hat mir mein liebes Eheweib hernach wohl zugeschlagen und mich lieb und werth gehabt. Und über das ich durch Gottes Seegen zu einer feinen Nahrung mit ihr kommen, hat sie mir 12 gesunde Kinderlein, die alle zur heiligen Tauff gelanget, uff diese Welt geborn, wie folget.

[Bl. 14] Verzeichnus meiner in der ersten Ehe erzeugten Kinder.

I. Den 4. Decemb. anno 1596 ward Nicolaus mein erster Sohn gebohrn, Sambstags früh zwischen 4 und 5 Horen, tauft ihn Herr Georgius Conradi, damals Capellan. Gevatter ward Herr 
M. Nicolaus Bauch, dazumahl Pfarrer zu Brait (band ein Goldgulden ein pro 20 Batzen und ein Carmen). Diss Kind verschied 12. Jul. anno 1597.

II. [fol. 33.] Den 23. Febr. 1598 Donnerstags abends vor Matthiae um 5. hor. ward unser zweiter Sohn Johann Wolffgang der erste dieses Nahmens geborn, ist aber nur 5 Wochen und 3 Tag alt worden (dann er 2. April Todes verfaren). Sein Tauffdot war Herr Johann Horn, freyherrl. Sainsh. Secretarius und kayserl. Notarius, ein herrlicher hochgelehrter Mann und trefflicher Redner. Taufft ihn Herr Valentin Apel, Pfarrer zu Northeim. Band ein 1 Ducaten pro $2 \mathrm{fl}$.

III. Den 11. Martii Sonntags Oculi anno Christi 1599 ward die erste Tochter Apollonia, die erste dieses Nahmens, abends zwischen 6 und 7 horen geboren, ihr Dot war Gevatter Anna Steinmezin, Müllerin zu Grassolzheim (weilen ich, wie folgen wird, unten damahls daselbsten Pfarrer war). Tauffts vorbesagter Herr Apel. Sie ist 3 Jahr alt worden weniger 1 Monat (dann sie den 13. Febr. anno 1602 verschieden) und konte überaus herrlich zu ihrem Alter beten im Catechismo und was man sonntäglich für die ganze Noth der Christenheit erhohlet.

IV. Den 25. Octobr. Sambstags früe vor Tags um 2 hor anno 1600 war die andere Tochter Anna gebohrn. Ihre Dot war vorwohlermeltes Herrn Secretarii Horns eheliche Haussfrau Anna (welche nach seinem Absterben hernach Anno 1604 27. Aug. mit Herrn Doctor Eisen, Vice-Canzler zu Onolzbach, Hochzeit (darzu ich auch geladen worden) gehalten).

Tauffts wiederum der Herr Apelt. 20 Batzen einbunden. Verschied diese Tochter selig 5. Decembr. Anno 1611 Vormittag um 8 hor an der Plag, betet stattlich und thut zuvor ein schōn Bekantniss, hat auch des Herrn Abendmahl kurz zuvor in der Kirchen sampt uns empfangen. Da die Wärterin sie in währender Schwachheit trōstet, sie würde wieder aufkommen, hat sie gesagt, nein; sie begers nicht, wann etwa der Vater stürbe, würde sie und ihre Mutter arme Witwen und Waisen.

[fol. 34.] V. Den 2. Aug. 1602 Abends zwischen 8 und 9 Horen ist die dritte Tochter Eva geborn Montags. Gevatter ward Hannsen Stollen Wirts Hausfrau Barbara (welche hernacher gen Archiv für Kulturgeschichte. 1 . 
Marckschönfeld [Stadt Scheinfeld] geheiratet zu ... Göttelbrünnern ..) 1 fl. eingebunden. Diss Kind ist $2 \mathrm{Jahr}$ alt worden weniger 8 Wochen, dann es ist Anno 1604 den 6. Junii ein viertel nach 1 hor gegen Tag verschieden.

Vl. Den 11. Junii 1604 Montags um 3 hor frūe ward gebohrn mein dritter Sohn, abermahl Johann Wolffgang II genannt, sein Dodt war Herr Martinus Nusshold Cancellist (so hernacher gräflicher Castellischer Secretarius und wohl beputtert) anstat und von wegen meines gnädigen Herrn von Sainsheimb, Herrn Johann Erckingers etc. taufft ihn Herr Apelt, wie auch voriges Kind, da dan vier Guldenstaler eingebunden worden. Verschied dieser Sohn seliger den 11. Novembr. Anno 1611 am Tag Martini im hohen Mittag an der Plag. Er ist schon in die Schul gangen gewesen und hat seine declinationes und coniugationes guter massen gekonnt, die 4 Coniugationes allmal zusammen lernen müssen, uff mein Verzeichnus, Amo, doceo, lego, audio, amas, doces, legis, audis etc. per omnia tempora et modos, wie man in [Bl. 14'] Schulen redt: hat ein statliches ingenium gehabt, und wann man ihm was von essender Speis nach üblichen Brauch zur Schulen mit geben, hat ers den armen Kindern geben, mit Fürwenden, er seine lectiones nüchtern lernen müste.

VII. Den 25. Aprilis Freytags zwo Stunden in der Nacht Anno 1606 ward die vierte Tochter Barbara I geborn. Ihr Tauffdot ward Barbara Valten Hilprands, eines Limburgischen Baurens (so hernach über dieselben Amptsbefohlene Schuldheiss worden) eheliche Haussfrau, tauffts Herr Barthol. Hoch, Ptarrer zu Dorff Cottenheim [Kottenheim], $2 \mathrm{fl} .2$ bazen eingebunden worden oder 2 Guldenstaler. Ist nur 5 Wochen und 2 Tag alt worden, 1. Jun. im Kindbett (wieder Verhoffen) verschieden.

[fol. 35.] VIII. Den 27. Sept. Sonntags 1607 ward meine fünfte Tochter Eva Barbara kürzlich nach 10 Hor in der Nacht geborn. Ihr Dot war das wohlgeborne Freyfraaulein, Frăulein Eva Barbara von Sainsheim (so hernach zu Herrn Philipps Ludwig Herrn zu Limpurg, Herrn Schencken Eberhardts zu Limpurg Sohn, sich ehlichen eingelassen). Tauffts $M$. Johann Postler, Apelts Nachfolger zu Nordheim. Diss Kind verschied den 7. Decembris Sambstags früe in puncto 3. horae anno 1611 in höchster Ge- 
duld und mit instāndigen fleissigen Gebet, ein recht frommes Kind, an Plag und Blattern und meint die gute Tochter selig, weil sie asse und die andern Kinder ausgeharret, auch eingenommen hātte, sie wolte diese Plag überwinden.

NB. Vergiss nit der traurigen Zeit, da mir inner Monatsfrist 3 liebe Kinder, darauf gute Hoffnung stunde, an der Plag starben, ja in 3 Tagen 2 Töchter begraben worden und so wenig leuthe mit zu Grabe giengen, ins Hauss aber fast niemand wolte, da doch ich im Sterben die Leuth fleissig besucht, das meine gethan und natürlicher weiss davon zu reden, das contagium davon ins Haus bracht: wiewohl mich Gott jederzeit wunderbarlich erhalten, weil mein Stūndlein noch nicht vorhanden.

IX. Den 4. August. 1609 Jahrs ward unsere VI. Tochter Barbara, die zweite dieses Nahmens geborn, Freytags frūe nach 8 Horen, einen Tag vor den vollen Mond. Ihr Dot Barbara Hilprandin vorgemelt (doch dazumahl Georg Zieglers, auch Limpurg. Schuldheisens ehliche Haussfrau). Tauffts M. Postler abends.

X. Den 18. Febr. 1611 Montags fráhe zwischen 2 und 3 Horen ward Apollonia, dieses Nahmens die andere und meine VII. Tochter, geborn. Gevatterin ward Anna, Hannsen [fol. 36] Gauterers, Wirths zu Grassolzheim [Krassolsheim] Haussfrau. Tauffts mein Bruder Georg Ludwig Ammonius, Pfarrer zu Jeckenheim, in Abwesen M. Postlers. War der Mond im Zeichen der Iungfrau und ein Tag verflossen nach dem Vollmond.

XI. Den 21. Novembris Sambstags anno 1612 ward meine achte Tochter Anna, auch die andere dieses Nahmens, zu Grassolzheim (wie dann alle meine Kinder ausser dem ersten und letzten) geborn, im Zeichen der Fische, einen Tag nach dem ersten Viertel zwischen 11 und 12 Uhr in der Nacht. Ihr Dot war Herr Caspar Leheleins, Sainsheimischen Haussvogts und Schuldheisens zu besagten Grassolzheim eheliche Hausfrau Anna. Taufft diese Tochter M. Postler, ist den 27. Sept. anno 13 abgewehnet worden.

XII. Den 14. Julii anno 1615 zwischen 7 und 8 Horen Vormittags an einem Freytag unter meiner Predigt ward mein vierdter Sohn, nochmahl Johann Wolfgang genannt, und also der III. dieses Nahmens geborn, im Zeichen des Krebs, einen Tag vor dem Neumond. Sein Tauffdot war Herr Hans Georg Wild 
Bürger und des Raths zu Marckbrait. Taufft ihn Herr Pfarrer Georg Conrad.

BBl. 15 Ist diss Kind nur $\%$ Jahr alt worden und den 14. Octobr. anno 16 in Beyseyn meines obgedachten Bruders, der ohngefehr darzukommen, (da es gegen Tag sehr kranck an Zāhnlein, isset doch) an diesen Tag gar sanfft verschieden [fol. 37], folgenden Tags begraben, vom Herrn Sebastian Lerleins Schreibern und Daniel Kellers Heimbecken Sohn hinaus zu Grab getragen worden. So viel sind meiner Kinder $10 \mathrm{zu}$ Grassolzheim und 2 zu Marckbrait bürtig, 4 Söhne und 8 Töchter.

Anno 1597 hat mein Haussfrau auch einen Bruch getragen, darüber sie sehr schwach worden. 11. Juni Anno 14 auch einen Bruch, so sich $\%$ Jahr verhalten, sich funden.

Nun komm ich wieder auf meine erste liebe Haussfrau selig, Apolloniam. Die ist $\mathbf{z w a r}$ auch, wie wir alle gewesen eine mangelhaffte oder gebrechliche Sünderin, doch hat ihr Gott wie auch allen Bussfertigen glāubigen Menschen um Christi Willen aus Gnaden verziehen, ihre Fehle bedeckt, vergeben und vergessen. Es hat aber die göttliche Majestāt diss Weibsbild mit so herrlichen Gaben und Tugenden gezieret, dass sie ein rechter Weiberspiegel gewesen.

Sie hat von Jugend auf fertig lesen und schreiben gelernet, auch bei ihrem teutschen Schulmeister etliche Species im rechnen begriffen, den Catechismum aus der massen gekonnt, Gott recht erkannt und gefürchtet und geliebet, streiff Vertrauen zu seiner göttlichen Majestaet durch Christum getragen, demūtig, gedultig gewesen, im Unglück unverzagt, gern gebetet, sonderlich im Habermann [fol. 38], Gott fleissig angeruffen und wiederum nach erlangter Hülffe gedanckt, aller Hexerey, Zauberey, Aberglauben von Herzen feind, sich vor Fluchen und Lästern gehütet, Gottes Wort gern gehört und geehrt, gefreuet, dass sie einen Geistlichen erheiratet, das Abendmahl des Herrn offt und viel genossen, das Predigtampt lieb gehabt, gern und fast mit allen Leichen gangea, über den Papstthum, da sie einsten zu Segnitz (B.-A. Kitzingen) die Wallfart gesehen und die Predigt gehört, sich erschüttert und ein schrecklich Missfallen ab derselben, wie auch anderer Secten Lehr getragen. Dem Nächsten die Werck der Lieb e:- 
zeiget, sich der nothleidenden angenommen, ihre Obrigkeit respectirt, ihrer Mutter und Freundschafft allen gebührlichen Willen erwiesen; ist aller Leichtfertigkeit und gegebenen Ergerniss von Herzen Feind gewesen, vertrāglich, kein Hass noch Neid getragen, freundlich mit jedermann, so viel sich leiden wollen, gehalten, aufrichtig denen Leuthen unter Augen gangen, jedem gesagt, was ihm zu sagen, er sey geistlich oder weitlich, unerschrocken, eine reine Jungfrau zu mir kommen, aller Unzucht und Linfläterey von Herzen Feind. Da sie einsten meiner Freund einer unter der Vesper in meinem Abwesen geküsst, hat sie mirs gleich, als ich heimkommen, eröffnet, und ist der Kerl bald darauf abgereist. Ich hab sie nie trunken gesehen, sie hat sich gar mässig gehalten, ist nicht mit losen Leuthen umgangen, schamhaffig gewesen, schlechte schwarze erbare Kleider getragen, jedermann das seine gerne gelassen, nichts entwandt, gar sehr sparsam gewesen, das ihre zu Rath gehalten, da ich lediger Weise mit meiner Cantoratbesoldung (so etwa des Jahrs uff $60 \mathrm{fl}$. werd in allen) mich allein nit betragen konte, ja noch etliche Schüldlein machte, hat sie mich von der Gesellschaft abgezogen und hernach zu einem feinen Aufnehmen bracht, [fol. 39] durch Gottes Seegen und mit ihrer fleissigen unverdrossenen Arbeit, dabey ich auch das meine nit gesparet, was ich gekont, thun und mir angestanden. Sie hat auch gerne geflickt und fein alles zusammengelesen, nichts vergehen lassen. Ist dem Borgen und entlehnen sehr feind gewesen, sie hett keinen Bissen Fleisch gessen oder Tropffen [Bl. 15'] Wein getruncken, wann er geborget were. Viel schöner Reimen aus der Hausshaltung Matthesii und sonsten hergenommen, zum rathsamen Leben dienstlich, zu erzehlen pflegen, sehr genau gewesen, doch zu ehren sich nichts an die Hand brennen lassen, meine Freund ja so lieb gehabt als die ihre, wann ich ihnen mit Leihen oder raisen gedient, sich nicht verwent gemacht. Hat sich genügen lassen, viel an ihr Sterbstündlein gedacht, mich herzlich geliebt und geehrt und hätte lieber selbsten Schaden und Krankheit ausgestanden, als mir solches zustehen gesehen, dem Gesind und Arbeitern gern essen gegeben und vor andern, wann sie Ehehalten oder Arbeiter bedorfft, bekommen können, genau gekaufft und redlich bezalt, nit viel lecker Bisslein kochen können, 
doch lezlich, wie wir sonderlich nach Brait kommen, etwas besser sich angriffen, Krebs und köstliche Speise, Hasen etc. nit gern kaufft, weilen das viel naschen bringt leere Taschen. Ist der Lügen Spinnen Feind gewesen, war nit schwãzig, loff nit viel in ander Leuth Häuser, hat die Kinder und Freund zum allerbesten angewiesen und ein recht Mutterherz gehabt, gern genehet und gesponnen, und was in Sprūchen Salomonis an 31 erfordert wird von Weibern, in der Wahrheit an ihr gehabt, und ich wüst nicht, an welcher Tugend ihrs gemangelt.

[fol. 40.] Diss hab ich also etwas weitlāufftigter, damit die nachkommenden Kinder und Freund sehen mögten, ich ihr nit vergessen, ob ich wol zum anciernmahl geheiratet, einführen wollen. Sie ist auch sonst erstlich schön und sauber gewesen und fein erbar regalisch anzusehen, einer feinen Länge, und ist vom alten Herm Grohen und andern verständigen Leuthen wohl gelobet worden.

Mit dem lieben Creuz ist ihr auch nicht verschonet, sie hat desselben einen guten Theil ausgestanden, sonderlich in Kindsnöthen ist sie eine harte Kindhaberin gewesen, und hătte meine Schwester Barbara das leztemal (dann je lānger, je strenger heissets da) nit das Beste bey der Sach gethan nechst Gott, so könnte sie ihr Kind wohl tod gehabt haben, dann sich eben die Kräffte sehr verlohren, und sie in ihren Leben nit viel Wein gebraucht und köstlicher Krafft gebender Sachen nit geachtet, hat auch das Fieber quartam meines Behaltens bey einen Jahr angetrieben. Ist fein gedultig in ihrem Creuz gewesen und hat gute Mittel zur Kranckheitzeit nit verachtet, sonderlich auf Herrn Apothekern Matth. Werdwein viel gehalten, der aus dem Urin sehr wohl getröstet, aher das Wiederspiel hat sich im Ausgang befunden, sonderlich in der Marterwochen Anno Christi 1617 (da ich ihr Mitwochs das heilige Abendmahl (weilen mein Herr Collega kranck war und sie keinen frembden Pfarrer haben wolte) uff Begehren und demüthige vorgehende Beicht und Glaubensbekänntniss gereichet). Darauf ist sle im Haupt etwas irr worden und doch gleich fein wieder zu ihr selbst kommen, hat fleissig gebetet, und dass ich ihr im Glauben dienen helffen wolte, angehalten.

[fol. 41.] Hat darauf ein sanfft seeliges Ende genommen 
(also dass, wo nicht das Kleine Humelein, wie mans nennt, mirs gesagt hätte, ich ihren Abschied ganz nicht gewust) actum 20. April vorgedachtes 17. Jahrs am heiligen Ostertag, da es 6 gegen Abend schluge.

Ist folgenden Dienstags 22. April ehrlich und solenniter zur Erden bestattet von M. Postlern, Pfarrern zu Erlach, der die Leichpredigt aus Rom. 14 gehalten in der Kirchen. Es ist auch ein ganzer erbarer Rath ausser Herm Kummern mit zu Grab gangen.

[B1. 16 Hat also meine liebe Haussfrau selig, immassen sie christlich gelebt, also auch christlich geschlossen und ein gut Lob bei männiglich hinterlassen. [fol. 41, 42.] Das Heurath- und Erbgut, so sie bekommen, belieff sich über $400 \mathrm{fl}$. [fol. 43.]

Nach verrichter meiner Apolloniae seligen Beschreibung komm ich nun wieder zu meinem Leben.

Als ich dann nun Cantor zu Brait gewesen vom 10. May anno 1595 an, hab ich mich so viel menschlich und müglich, treulich dienen gebraucht, dem Reichen gethan wie dem Armen, hab aber schrecklichen Undanck und viel Trübsal erfahren und ausgestanden, so ich nit schreiben mag.

Anno 1597 18. Jul. ist mir die Pfarr Grassolzheim, so 3 Stund Wegs von Winssheim (Windsheim im B.-A. Uffenheim) liegt, angetragen und ins Hauss angeboten worden aus gn. Herrschafft Befehl durch Herrn Nicolaum Grohen, Schuldheisen zu Marckbrait.

Folgenden Tags bin ich zum Seehaus von dem wohlgebornen Herrn, Herrn Georg Ludwig von Sainssheim etc, zum Pfarr gesagtes Orts angenommen, in Beyseyn Herrn Valten Apelts, Pfarrers zu Northeim, deme ich recommendirt worden, und hab so balden mein Priesteriurament ihrer Gnaden geleistet, 19. Jul.

In diesem 1597. Jahr 24. Jul. bin ich zu Marckbrait ordinirt ix. Trinitatis und hab Nachmittag die Epistel zur Probpredigt gethan. Darauf folgenden Tag mit meinen Herrn Collegis mich gelezet, den 26. diss mit meiner Apollonia die neu Pfarr besehen. Den 28. darauf uffgezogen mit meinem Schwagern Weib, Mutter und Geschwistrigten und die Pfarr im Nahmen der Heiligen Dreyeinigkeit versehen, von der Verstöhrung Jerusalem erstes mal geprediget. Dieweil aber diese Pfarr vierdtes Theils Limpurgisch, 
welches [fol. 44] ich anfangs nicht gewust, und die Limpurget ihr recht $\mathrm{zu}$ erhalten mich nicht investiren helffen wolten, ich liess mich dann zuvor in ihrer Herrschafft examiniren, als hab ich nach Obersundheim [Obersontheim in Württemberg] derenwegen verreysen und mich examiniren lassen müssen uff meiner Costen. Herr Paedianus Pfarrer daselbst und der Pfarrer zu Mittelfischach haben mich vorgenommen in Beyseyn Herrr Secretarii und allererst von der Formula Concordiae und stritiger: Articuln gehört über die 2 Stund. Darauf ein gut Testimoniurr nach Speckfeld [B.-A. Scheinfeld] zu bringen ausgefertigt und mich zur Tafel geladen, da ich mit Herrn Schencken Alberten und seinem Gemahl unter andern gessen. Vom 23. Sept. biss 27 . hab ich die Reise verrichtet und ist dennoch die investitur, weilen die rothe Ruhr zu Grassolzheim regiert und sonsten die Herrschafft andere Geschäfft gehabt, bis uff den 3 ten Sonntag nach Oberstag anno 98 verschoben worden 29. Januar.

$\mathrm{Da}$ ich dann bederseits Beampten, Sainsheimischen Herrn Secretario Johann Horn und Herrn Christoph Hohenbergern, Limpurg. Amptmann, wegen beeder Herrschafften noch eine Pflicht leisten müssen, darauf die Predigt gehalten und ein stattliche tractation mitgenossen; dem actui haben ihrer viel beygewohnet, Herr Valten Apel, Pfarrer zu Nordheim, und seir: Schulmeister Bürlein, Herr Bartholom. Hoch, Pfarrer zu Dorif Cottenheim, [Kottenheim im B. A. Scheinfeld], Herr Doctor Juris Burcard von Winssheim, Jeremias Junckher, Herr Secret. Schreiber, so hernach Pfarrer zu Deitenheim [Deutenheim bei Scheinfeld] worden etc.

4. Jun. anno 1606 bin ich, weilen Herr Apelius, voriger Winssheimischer Decanus, [fol. 45] Todes verfahren, uff vorgehende mir unbewuste Deliberation Herrn Hofmanns, Oberrichters zu Winssheim, (der über den ganzen Rath daselbst zu commendiren hat und sein Lehen vom Kayser empfangen) und Gutachten Herrn Andreae Nagelii, Pfarrers zu Winssheim, und des Ministerii daselbsten einstimmen von meinen Capitelsbrüdern zum Decano desselben würdigen Capitels angenommen, und sind mir ihre fundationes, privilegia und Einkommen gewiesen, auch eine statliche Malzeit gehalten worden. 
[Bl. 16] Habe darauf wie die vorigen Jahre, also auch hinfort jahrlich uff Mitwochen vor Pfingsten diss Capitul zuweilen mit Leibesgefahr besucht und einen locum oder Stück und articul der heiligen Schrifft vorgenommen nach dem andern.

Anno 1611 bin ich zu Grassolzheim in mein eigen Hauss, so Limpurgisch Lehen, uff gn. einwilligen meines gn. Herrn von Sainsheim eingezogen, damit der Schulmeister im Pfarrhaus wohnen könte.

Anno 1614 11. Januar, da mein gnädiger Herr, Herr Johann Erckinger von Sainsheim zu Grassolsheim gejagt und meinen Vorfaren Wilhelmum Wisnerum [1610-1614] abzuschaffen Willens, gewisser Ursachen halber, beut Er mir, in Beyseyn Herrn Philipp Ludwigs von Limpurg, auch Georgii Kummers und Daniel Oertels, Notarii und Schulmeisters zu Breit, selbige Caplaney an, wann sie verlediget. Ob ichs nun wohl anno 160225 . Januar, 3. und 11. Febr. vorhin abgeschlagen, da mirs auch durch etliche des Raths und Predigampts zu Brait [fol. 46] angetragen und Mittel gezeiget worden, Jedoch hab ich dissmal, da Wisnerus erlassen und meiner begert worden, endlich aus vielen Ursachen eingewilliget, wie aus nachfolgender Copia zu ersehen.

Denen Erbarn und Ehrsamen unsern lieben getreuen Burgermieistern und Rath zu Marckbrait.

Johann Erkinger von Sainsheim, zu Hohen Cottenheim, Seehaus, Sinchingen und Erlach etc. Freyherr.

Unsern gn. Gruss zuvorn, Erbar und Ehrsame liebe Getreue, was wir euch in ernstlichem Befehl wegen Renovirung und Fortschaffung eures nunmehro gewesenen Caplans Wilhelm Wisers, sodann auch daneben vertröster Praesentation und Verordnung einer andern mehr qualificirten und wirdigern Person neulichst uberschrieben und uffgetragen, dessen habt ihr euch guter massen zu erinnern. Dabey solchem Beschaid wir nun gewisser erheblicher Ursachen willen endlich zu beharren gedenken und entschlossen. Solchem unsern Zuschreiben und Beschaid nun gemess thun wir zu obberegten nunmehr vacirenden Diaconat oder Caplaney Krafft habenden Juris Patronatus supremi praesentiren gegenwertigen 
wirdigen und wolgelerten unsern Pfarrern zu Grassolzheim Wolfg. Ammonium, als welchem wir dieselbe um seiner uns bewusten genugsamen qualitaet, und weilen sein Vater seeliger des Orts christlicher gemein und Schulen wohl vorgestanden und solcher Er auch wohl thun kann und will, [fol. 47] wie uns nit daran zweifelt, allbereit würcklich conferiret, also und soicher gestalt, dass derselbe $\mathrm{zu}$ solchem Diaconat und Caplaney inzwischen Ablauff 2 Monaten der christlichen Gemein und Kirchen zu Marckbrait vorgestellt, investirt und eingesezt, immittelst aber solcher Zeit die zu solcher Caplaney gehörige Wohnung geräumet und dem abgeschafften Wiser sein Gelegenheit anderstwo zu suchen von Euch angesagt und verfügt werden soll. Das zu geschehen wir uns abermahls verlassen, denen wir sonsten mit gn. Willen wolgewogen verbleiben. Datum Seehaus den 8. Febr. anno 1614. Darauf den 27. Febr. diss 14. Jahrs bin ich in Beyseyn meines Herrn Collegae und M. Johannis Postleri, damahls Pfarrern zu Northeim, auch Herm Marci Schechsii, Pfarrers zu Erlach, vom Herrn Secretario investirt worden, da von eines erbarn Raths wegen Herr Lerlein und Herr Conrad Hartung bey, war der Sonntag Sexagesima, M. Postler predigte uff gn. Herrschafft [Bl. 17] Begern; am Tag Matthiae zuvor, hab ich zu Brait meine Probpredigt gethan aus derselben Historia Actorum 1. Darauf eine Malzeit eingenommen in Georg Wirthshauss.

Man hat mir aber vorigen Abends, als Herr Pfarrer und ich in gedachtem Wirthshauss die Abendmahlzeit gehalten, vor essens durch Herrn Secretarium Philipp Schattemann vorgehalten, ich solte alle Tag ein Stund oder 2 Schul halten helffen und darinnen laboriren, aber mein Herr Collega hat solches wiedersprochen und nicht darzu verstehen wollen. Gott vergelt ihm.

[fol. 48] Hernach sind mir nachfolgende Puncten, darauf ich folgendes Tags mein Handgelübt gethan, in der Schul (allda mir auch die Praeceptores Daniel Oertel und Adamus Rab als Schulmeister (wie mans damals genennt) und Cantor angelobt haben vor der Predigt) zu Gemüth geführet worden 26. Febr. anno 14.

1) Augspurgische Confession anno 1530 übergeben und Lutheri Schrifften soll ich mir lassen befohlen seyn und nichis in Ceremonien ohne der Herrschafft Wissen ändern. 
2) in Schul und Kirchen die Jugend fleissig unterrichten helffen.

3) uff die Schuldiener Inspection halten.

4) Pfarrern respectiren.

5) Heiligen Wandels mich fleissigen.

6) gn. Herrn von Sainsheim etc. für den einigen Collatorem halten.

Von dieser Zeit an hab ich die Caplaney zu Brait mir, wie billig, lassen angelegen seyn. 14. Mart. zu Seehaus valediret und gesegnet und darauf den 15. Mart. diss 1614. Jahrs bin ich mit allen den meinen gen Marckbrait von Grassolzheim ausgezogen mit 6 Fuhren und 30 fl. par Geld und etlichen dn., und haben nich meine alte Pfarrkinder ungern verloren, ja alle Männer auf der Porkirchen, da ich meine Lezpredigt gethan, geweint und keinen gewissen Pfarrer gehabt, biss uff 29. May Herr Johann Cuppelich (welcher 6. Jul. anno 74 unsers Herrn Pfarrers Georgii Conradi Tochter durch den [fol. 49] gnädigen Herrn und mich Werbende, erfreyet) allhie in Beyseyn hochwohlgedachtes gn. Herrn und eines Calvinischen Herrn von Schwarzenbergs [im B. A. Scheinfeld] allhie ordinirt worden, so an mein stat kommen. In diesem Flecken Brait hab ich nun seiter gedachter Investitur nit w'enig ausgestanden von des Wisers Freunden und Grohischen, dann der alte Schuldheiss Groh damahls sowohl als der Wiser abgesezt worden. $\mathrm{Er}$ ist aber hernach in sein Ampt wieder eingewiesen und restituirt, und hab ich durch Gottes Gnad überwunden, bin in meinem Beruff unerschrocken gestanden und hab meiner Feind etliche sehen das Land zeitlich räumen.

[fol. 107] Als mein Collega Herr Georg Conrad Pfarrer 25. Aug. anno 31 verschieden, und ich Ihne solte den 28. diss bestatten und die ordinari Predigt darzu hatte, kan ich in der Nacht nit schlaffen, fâllt mir ein, ich soll gen Segniz zu Doctor Göring gehen und seines Raths leben, dass ich nicht hinter der Pfarr hingehe. Dann ich nit gern vor Herrn Pfarrers Begräbniss ansuchen wollen. Uff den Tag geh ich nach Segniz, uff den Weg räth mir Herr Wildmeister, ich soll ein Supplic an gn. Herrn stellen, man hab von einen successore allbereit geredt, der morgen Sonntag predigen soll. Herr D. Göring liegt im Schweiss, lest 
mir sagen, er wiss nichts, lest mir doch sagen, ja schreibts auch, wan er als Rath gefragt werd, mein Bestes zu befördern. Im Heimweg sprechen mein Herr Wünderlein und Wildmeister zu, lch soll nit verziehen mit meiner Supplication an gn. Herrn, Herr Schuldheiss lesst mir uff Bitt ein E. Rath zusammen fordern, die bitten für mich; ich hab an Herrn Secretar. Wolffium, wie auch an E. E. Rath [Bl. 17'] und Doctor auch geschrieben. Gott der allmächtige hat mein Gebet erhört. Der Bott ist eben Ihrer Gnaden in die Hand kommen, so ausreiten wollen, und mir der Pfarrdienst zugesagt worden, obs wohl dem M. Salom. Blechschmidt [aus Hof, Caplan 1632], der hernach mein Collega worden, z.weymahl versprochen gewesen, und ist mir das Decret, als ich von Herrn Pfarrers seligen Leichpredigt müd und matt heimkommen, von Herrn Johann Merck, Gerichtsschreibern, gebracht, der dem Herrn Selden vorkommen; darauf tangen die Glückwünschungen an und den 4. Sept. bin ich zur Pfarr, M. Blechschmidt zum Diaconat von Herrn Clemente Gundermann, Pfarrern zu Northeim investirt, und als ich diesen Collegam etwa $5 / \%$ Jahr gehabt, wird nach seinem Absterben $M$. Johannes Cranz, Rosenbergischer Pfarrer zu Waldmannshofen, [im O. A. Mergentheim] mein Collega. [Caplan 1632, Pfarrer in Marktbreit 1634-1645.] [fol. 49. Als ich nun 3 Jahr und etliche Wochen wiederum; zu Brait gehauset und gedienet, stirbt mir, nach dem zuvor hingegebenen Kinde, davon oben im 36. Blat, auch mein liebes Weib (wie im 41. Blat zu ersehen), welche von Mariae Verkündigung biss uff den 2. Sonntag nach Ostern gelegen, eben zu der Zeit, da mein Herr Pfarrer krank und die Kirchenarbeit in der Marterwochen uff mir allein lag (immassen ich dann inner 7 Tagen 8 Predigt gethan, durch Gottes Gnad, und sehr viel Beichtkinder. ja am Ostertag früe noch 40 frembde Personen aus dem Pabstthumb Ehehalten gehöret. Hab ich also eine rechte Marterwochen gehabt. Gott der allmächtige aber, der nach dem Ungewitter die Sonne wieder scheinen lässet und nach dem Heulen und Weinen die Seinen mit Freuden überschüttet, hat meinen Sack ausgezogen, mich mit Freuden gegürtet und mir meine Klage [fol. 50 in einen Reyen verwandelt. Wie Tobiae am 3. und Psalmo 31 geschrieben stehet, das ist, Gott der Herr hat mich nach ausgestandenen 
Wittib Stande durch M. Postlers und seines Weibs Unterhandlung zu der andern Ehe befördert und anderweit erfreuet.

Dann als ich meine gewesene Haussfrau selig alle Tag sowohl über essens und wann ich allein war, als bei den Leuthen beweinete und nirgend auskam, M. Postlers Weib aber zu Ochsenfurt den Schwager Gabriel Hartmann angeredet, Er solte mich heissen je zuweilen ausspazieren und zu Ihren Herrn, der damahls zu Erlach Pfarrer war, kommen, hab ich der Sachen nachgedacht, dass mit Trauern kein Toder herwieder zu bringen wäre und ahne das mein Herr Collega mit einem greinenden Weib allhie Schreinerin mich verglich etc. Und demnach bald darauf der Tag einen mich uffgemacht nach Erlach, in Willens, gedachten Postler zu besuchen. Da nun ich eben gegen dem Mittag in gröster Hiz ausgangen, und der Pfarrer nit zu Haus war, sein Weib aber mir alle Ehr thăte mit gutem starcken sechzehener Wein, derselbe mir im Durst getruncken in Kopff schlug, das Weib aber mich, wann meine Zeit aus wäre, wohl zu versorgen getröstete und mir Herrn Pfarrern von Sommerhausen und seine Tochter Reginam in bester Form mir commendirte und sagte, es wãre ein kleiner Weg dahin, Ich auch vorhin des guten Herrn Person wohl kante von Seehaus her und sein Kunst wusste und M. Postler ohne dass nit zu Hauss und mir die Weil lang dabey war, lasse mich bereden und spaziere mit nach Sommerhausen, vom Jungkherrn Fronhöfern [fol. 51] und seinem Weib, auch der Pfarrerin begleitet, da mir dann alle Ehre wiederfahren, auch, als ich mich unversehens geschnitten, mit gegebenen Verbindung uffs schōnest mit mir gebaret worden. Darauf sind wir wieder nach Erlach gereist und bin ich die Nacht [Bl. 18] daselbst blieben.

Morgens, als mich M. Postlers Haussfrau fragt, wie mir die Jungfrau gefiele, und ich sie nit verachten konnt, will ihre Wäscherin mich verhezen und Hundshaar drein hacken: aber von Gott beschert, bleibt unverwehrt. Ich warte meines Dings und lass es den lieben Gott walten. Uff Johannis Baptistae Tag hat gemeldte Tochter Regina einen schönen verguldten Cranz uff ihres Vatters Befehl gemacht, damit mehrbesagter Postler ist angebunden worden, der wird mir Sonntags nach Johannis an unsern Marckt neben der Person wiederum trefflich commendirt. 
lst mir auch noch nit ausgefallen, dass $M$. Postlers Weib bey Ihrer Seelen Seeligkeit beteuerte, wann ihr Herr ein Wittwer were, sie demselben kein ander Weib als diese Reginam, wann sie wolte, wünschen thete, um ihrer vielen Tugenden und gutens Hausshaltens Willen. Darauf als M. Postler sich zu lösen verspricht und meiner nach etlichen Tagen darzu begehrt, ich auch seinem Verheisen nach der Jungfrauen bey solchem Convivio erwarte, eheliche Kundschafft zu machen, und wann meine Zeit zu traueren aus wäre, mich zu bewerben, kommt sie nicht, sondern nur ihre Eltern, und hats Postler weiter als ich begehrt und mit lhm abgeredt, [fol. 52] gespannet; kommet Herr Statvogt Samuel Man, von Herrn Pfarrern zu Sommerhausen beschrieben, darzu. M. Postler, von mir übel angefahren, dass er vor der Zeit so weit lossschlüge und mir böse Nachred verursachte, ändert den stylum und bittet, wenn meine Zeit aus wäre und ich ansuchte, keinen Korb zu geben. Der Regina Herr Vater hat etwas Bedenkens, weil ich Kinder hab und er nit viel Heuratguts hab und was des Dings mehr, aber Herr Statvogt redt mein Bestes, mir ist alles wohl zufrieden. Als wir nun über der Mahlzeit unter der Linden sizen, kommt ein erschrecklich Wetter, die Pfarrerin will heim zu ihrem saugenden Kind, der Herr bleibt zu Erlach. Ich gedencke, ich mögte die Person wohl noch einmahl sehen, begehre demnach gedachte Frau Pfarrerin heimzubegleiten im grossen Wetter und Regen. Da ist mir aller guter Will wiederfahren und hab ich Gottes Schickung sonderlich gespūhret und demnach dahin, wo Herr Schwager Hübsch mich gern gesehen und befördern wollen, nit gelangen können noch sollen. Folgenden Morgens bin ich wieder von Erlach kommen, hab referirt, und sind wir uff den Abend (dann ich mich bereden lassen) wieder nach Sommerhausen mit gesamleten Rath, wie man sagt, gelanget, und haben derselb Herr Pfarrer und ich als Vater und Sohn, einander angenommen gemeiner Weise, doch ist etwas mit untergelauffen von Tractation, wann meine Zeit aus wäre, und wir mein Hausswesen besehen hātten.

Mittlerweil als ich heimkommen und etliche Tag fürüber, stell ich auf und forsche nach, was die Jungfrau für ein Lob hab, Jedermann aber sagt ihr alles gute nach, und hat sonderlich ihre 
liebe Mutter selig (wie aus der Leichpredigt Herrn Nagelii, Pfarrers zu Winderhausen, zu ersehen) ein [fol. 53] stattlich Lob und Zeugniss von ihren guten Sitten.

15. Julii diss 1617. Jahrs kommt der Herr Pfarrer von Sommerhausen mit seinen beden ältesten Töchtern und Tochtermann, und besehen mein Hausswesen, da spüret sich die Freundschafft an, sonderlich des folgenden Tags 16. Julii, und bin ich weiterer Heirats Sorg abkommen. Diesen Abend Herr Pfarrer hie und sein Weib, M. Postler und sein Weib, Herr Schweher samt seinen Geferten, ich und die Braut gen Frickenhausen [B. A. Ochsenfurt] zu Wasser gefahren, $1 \mathrm{fl}$. verthan.

Darauf den 28. Julii diss 1617. Jahrs hab ich mich mit meinen Kindern gesezt, das ist, [Bl. 18'] ich hab in Beyseyn Herrn Sainsheimischen Schuldheissens Grohen von Herrschafft wegen, Herrn Johann Hübschens, Pfarrers zu Sickershausen [B.A.Kitzingen], von der Kinder wegen und Freundschaffts halber, Herrn Pfarrers Georgii Conradi, Herrn Sebastian Lerleins, Notarii und Gerichtschreibers, mich verglichen, was ich jedem Kind zum Vorauss geben solle und wolle, wie alles in Heiratsnotul zu finden. Da daselb vollendet, hab ich in Gottes Nahmen bey Wesende Ihres Vaters Sie zur Ehe genommen und biss uff fernerer Priestershand durch Herrn Pfarrern an die Hand geben lassen.

Warum ich aber so balden wieder geheiratet, sind folgende Ursachen zu merken:

1. Die Kinderzucht war mir, der nit alle mahl zu Hauss, allein $z \mathrm{u}$ schwer.

2. Das Studiren war schlecht um der Gesellschafft willen, die mich anloffe.

3. Melancholey war gross und sonsten Gefahr Kranckheit halben, darinnen einen niemand besser als sein Weib warten kann.

4. Mein Gut kam ins abnehmen, deren sich zuschlagenden Leuth halben.

5. Die Wärterin (weilen ich kein Magd oder Jungmensch, Argwohn zu verhüten, leiden kont) costet mich wöchentlich ein Ort, ohn essen und trincken [fol. 54], so gut ichs genossen.

6 . Hab vorhin schon eine Tragoediam gesehen am kleinsten Kind, als ich heim kam von Sickershausen [bei Kitzingen] von 
Herrn Hübschen, den ich an Johannistag angebunden, dann das Kind, als die Wärterin wieder meinen Befehl zu den Arbeitern ir: die Mainleiten gangen, war daselbst herab vom schmalen Weg gekugelt und lauter Blut worden, von der Scheuin aufgefangen. dass es nit gar in Main gehergelt, und von der Rectorin Juni $i$ Weib abgewaschen worden.

7. Meine Braut hatt mehr Freyer, wo ich nit zugriffe, und sonderlich einen reichen Weissgerber von Schweinfurt, auch einen Pfarrer von Hoheim [bei Kitzingen], und war ich mit den dreyen Töchtern Ihrer Köpfe halben (darüber die Wärterin offt samt den Kindern weinete) nit jedermanns Kunff.

8. Ein Eheweib haltet eher etwas zu Rath dann eine Wärterin. Ich will jetzt geschweigen, wie ich ungeachtet fleissiges Versperrens dennoch viel verlohrn und doch niemand nichts zu zeihen noch überweisen weiss.

Den 25. Aug. diss 1617. Jahrs sind Herr Balthasar Held, des Raths allhier, und Adam Rab Cantor mit mir nach Frickenhausen gefahren und haben die Braut und frembde Hochzeitleuthe angenommen und empfangen, die Ochsenfurter Schüzen aber haben auch viel Freudenschüss vor und nach gethan und derowegen ihre Ergezlichkeit empfangen vom Herrn Schwehervater.

[fol. 55] Darauf folgenden Tags, Dienstags nach Bartholomaei 26. Aug., ich meinen andern Kirchgang und hochzeitliches Freuden- oder Ehrenfest gehalten mit meiner lieben Regina. Es haben aber uns neu angehende Eheleute zu unsern hochzeitlichen Elırentag gedienet und ohne wir beede zur Kirchen mitgangen 67 Personen, unter welchen die merckwürdigsten sind Herr Nicolaus Grohe, Schuldheiss zu Marckbrait, und als meines gnädigen Herrns von Sainsheim Gesandter. Herr M. Hieronymus Theodoricus Schwehervater. Herr Georgius Conradi, Pfarrer allhie zu Marckbrait. Herr Sebastian Lerlein, Schwager und Gerichtschreiber allhie. [BI. 19] Herr Matthias Engelhardt, Schwager und des Raths allhie. Herr M. Thomas Jung, Pfarrer zu Sundheim [Mönchsondheim im B. A. Scheinfeld], Vetter.

Herr Gabriel Hartmann, Schwestermann mir zu Kirchen Herr Conrad Hartung, des Raths allhier Imitgangen. 
Herr Johann Hübsch, Pfarrer zu Sickershausen, Schwager. [fol. 56.]

Herr Georg Hager, des Raths zu Kitzingen, der jetzigen Schwieger Stieffvatter.

Herr M. Johann Postler, Pfarrer zu Erlach.

Herr Hanns Georg Wild, des Raths allhier, ein Gevatter.

Herr Balthasar Widmann, Fürstlich Brandenburgischer [Wein-] Bergmeister zu Sommerhausen, ein Schwager.

Herr Christoph Hohenberger, der Braut Schwester Mann.

Herr Balthasar Held, des Raths allhier.

Herr Adam Rab, Cantor allhier.

Herr Peter Planck, teutscher Schulmeister allhie.

Weiber oder Frauen:

Frau Dorothea Körnerin, Kellerin.

Frau Barbara Hohenbergerin, Braut Schwester.

[fol. 57] Frau Margaretha Conradin, Pfarrerin allhier.

Frau Margaretha Gröhin, Schuldheisin allhier, nur zur Kirchen.

Frau Pfarrerin von Mönchsundheim.

Frau Pfarrerin von Sickershausen.

Frau Pfarrerin von Erlach.

Frau Bergmeisterin von Sommerhausen.

Frau Schulmeisterin von Sommerhausen [Margaretha Gast, vgl. Caspari, Der Schulmeister und sein Sohn, 1853].

[fol. 58] Frau Plänckin, deutsche Schulmeisterin. [fol. 61.]

Es hat mir aber mein lieber Herr Schwehervater funfzig Gulden an guten alten herrlichen Reichs Talern, deren jeder 21 Bazen golten, zum Heuratgut vor der Hochzeit erlegt, da ich mit die Hochzeit verlegt und Kleider zu Kitzingen erzeuget.

Ich hab auch von ihren Anherrn oder Altvattern Herrn Zacharia Bechtern, gewesenen Pfarrern zu Feldsteten, nach seinen Tod anno $20 \mathrm{im}$ Majo bekommen und ererbet [fol. 62] an Mobilien über meinen Abgang um ein rechtes taxirt, so gut als $30 \mathrm{fl}$.

[fol. 67] Das Leben meiner andern Hausehre Reginae.

Ihr Vatterland ist Sommerhausen, 3 Stund Wegs von Würzburg, der Herzoglichen Stadt in Francken gelegen. Sie ist aber Archiv für Kulturgeschichte. I. 
den 27. Aprilis anno Christi 1597 geborn. Ihr lieber Herr Vater ist der Ehrenwürdige und Hochgelarte Herr M. Hieronymus Theodoricus, Limpurgischer Pfarrer zu besagtem Sommerhausen. Ihre liebe Mutter (selig) war Frau Regina Bechlerin, des auch Ehrwürdigen und Wohlgelahrten Herrn Zachariae Bechlers, Pfarrers zu Feldsteten selig (so von Augspurg mit Herrn Doctor Georg Müllern ${ }^{1}$ ) und andern Kirchendienern vertrieben und verfolget worden) [vgl. Paul von Stetten, Gesch. der Stadt Augsburg 1, 696] und Apolloniae vom Geschlecht einer Dempflerin Tochter.

Ist von Kindsbeinen an und in der zarten Jugend nach Pauli Vermahnung zur christlichen Schul gehalten, unter Herrn Johann Weichselio, welcher hernach zu Lindelbach [bei Ochsenfurt] und Westheim [bei Kitzingen] Pfarrer und dess Orts ein Gevatter worden, meines [BI. 19'] Herrn Schwehers, nemlich ....

Die Hausszucht ist auch gut gewesen, welche viel bey der Sachen gethan, darum sie dann nicht nur das gedruckte, sondern auch geschriebenes fertig lesen und wohl schreiben gelernet, auch der Gottesfurcht sich vor allen Dingen geflissen und [fol. 68] viel schöner Psalmen sampt den Feyer- und Sonntäglichen Evangelien, auch schöne Gebet allerhand auswendig recitiren können, und ist dannoch darneben zur Haussarbeit, neen, stricken und andern weiblichen Verrichtungen uffs Beste von ihren Eltern und alten Schuldheisen angewiesen, auch fleissig im Haus behalten und wenig ausgelassen worden.

(Schluss folgt.)

1) Vgl. Beitr. 7, 4. Dr. Georg Müller (Mylius) war 1548 in Augsburg geboren und leiste:e der Katholiken auf das entschiedenste Widerstand, bis er sich endlich zur Flucht aus seiner Vaterstadt genötigt sah. 